\title{
RTTOV-gb - adapting the fast radiative transfer model RTTOV for the assimilation of ground-based microwave radiometer observations
}

\author{
Francesco De Angelis ${ }^{1}$, Domenico Cimini ${ }^{2,1}$, James Hocking ${ }^{3}$, Pauline Martinet $^{4}$, and Stefan Kneifel ${ }^{5}$ \\ ${ }^{1}$ CETEMPS, University of L'Aquila, L'Aquila, Italy \\ ${ }^{2}$ IMAA-CNR, Potenza, Italy \\ ${ }^{3}$ Met Office, Exeter, UK \\ ${ }^{4}$ Météo France - CNRM/GAME, Toulouse, France \\ ${ }^{5}$ Institute for Geophysics and Meteorology, University of Cologne, Cologne, Germany
}

Correspondence to: Francesco De Angelis (francesco.deangelis1@graduate.univaq.it)

Received: 23 March 2016 - Published in Geosci. Model Dev. Discuss.: 9 May 2016

Revised: 14 July 2016 - Accepted: 27 July 2016 - Published: 19 August 2016

\begin{abstract}
Ground-based microwave radiometers (MWRs) offer a new capability to provide continuous observations of the atmospheric thermodynamic state in the planetary boundary layer. Thus, they are potential candidates to supplement radiosonde network and satellite data to improve numerical weather prediction (NWP) models through a variational assimilation of their data. However in order to assimilate MWR observations, a fast radiative transfer model is required and such a model is not currently available. This is necessary for going from the model state vector space to the observation space at every observation point. The fast radiative transfer model RTTOV is well accepted in the NWP community, though it was developed to simulate satellite observations only. In this work, the RTTOV code has been modified to allow for simulations of ground-based upwardlooking microwave sensors. In addition, the tangent linear, adjoint, and K-modules of RTTOV have been adapted to provide Jacobians (i.e., the sensitivity of observations to the atmospheric thermodynamical state) for ground-based geometry. These modules are necessary for the fast minimization of the cost function in a variational assimilation scheme. The proposed ground-based version of RTTOV, called RTTOV$\mathrm{gb}$, has been validated against accurate and less time-efficient line-by-line radiative transfer models. In the frequency range commonly used for temperature and humidity profiling (22$60 \mathrm{GHz}$ ), root-mean-square brightness temperature differences are smaller than typical MWR uncertainties $(\sim 0.5 \mathrm{~K})$ at all channels used in this analysis. Brightness temperatures
\end{abstract}

(TBs) computed with RTTOV-gb from radiosonde profiles have been compared with nearly simultaneous and co-located ground-based MWR observations. Differences between simulated and measured TBs are below $0.5 \mathrm{~K}$ for all channels except for the water vapor band, where most of the uncertainty comes from instrumental errors. The Jacobians calculated with the K-module of RTTOV-gb have been compared with those calculated with the brute force technique and those from the line-by-line model ARTS. Jacobians are found to be almost identical, except for liquid water content Jacobians for which a $10 \%$ difference between ARTS and RTTOV-gb at transparent channels around $450 \mathrm{hPa}$ is attributed to differences in liquid water absorption models. Finally, RTTOVgb has been applied as the forward model operator within a one-dimensional variational (1D-Var) software tool in an Observing System Simulation Experiment (OSSE). For both temperature and humidity profiles, the 1D-Var with RTTOVgb improves the retrievals with respect to the NWP model in the first few kilometers from the ground.

\section{Introduction}

The planetary boundary layer (PBL) is the single most important undersampled part of the atmosphere (National Research Council, 2008). While the thermodynamical state of the atmosphere is well measured at the surface by ground 
in situ sensors and in the upper troposphere by satellite sounders, there is currently an observational gap in the PBL. According to the WMO Statement Of Guidance For Global Numerical Weather Prediction (WMO, 2014), there are four priorities for atmospheric variables not adequately measured in the PBL: wind profiles, temperature and humidity profiles in cloudy areas, precipitation, and snow mass. Ground-based microwave radiometers (MWRs) provide temperature and humidity profiles in both clear- and cloudy-sky conditions with high temporal resolution and low-to-moderate vertical resolution, with information mostly residing in the PBL (Cimini et al., 2006). Ground-based MWRs offer to bridge the current observational gap by providing continuous temperature and humidity profiles in the PBL. When combined with satellite observations, the total information content of the derived atmospheric profiles can be significantly enhanced (Ebell et al., 2013). The data assimilation (DA) of MWR observations into numerical weather prediction (NWP) models may be particularly important in nowcasting and severe weather (fog, convection, turbulence, etc.) initiation. The assimilation of MWR data has been recently investigated (Cimini et al., 2014; Caumont et al., 2016), assimilating temperature and humidity profile retrievals from a network of 13 MWR members from the international MWRnet network (Cimini et al., 2012). Results showed neutral-to-positive impact. However, these experiments used retrieved variables (temperature and humidity profiles), whereas the assimilation of raw measurement (TBs) is found to have more impact on the NWP forecasts in the case of satellite data (Geer et al., 2008).

Accordingly, a potential way to increase the impact of MWR DA is to assimilate measured radiance (or brightness temperatures, TBs) directly instead of retrieved profiles. With this type of assimilation, all the degrees of freedom for signal of MWRs (Löhnert et al., 2009) can be used to improve the NWP model forecast in the PBL. In order to assimilate $\mathrm{TB}$, a radiative transfer (RT) forward model is needed. The RT model allows the TB to be computed for selected radiometer channels based on the NWP model state vector. TB differences between the modeled and measured observations can be used within a variational scheme (Courtier et al., 1998) that takes the corresponding uncertainties into account to retrieve temperature and humidity profiles in the first few kilometers from the ground, where MWRs provide the maximum information content. In addition, the Jacobians (i.e., partial derivatives with respect to the state vector) of the radiative transfer model are required to minimize the distances of the atmospheric state from both the first guess and the observations in a variational data assimilation process. These Jacobians represent the sensitivities of observations to the atmospheric thermodynamical state.

The fast RT model RTTOV (Radiative Transfer for the TIROS Operational Vertical Sounder (TOVS)) is widely used to simulate radiance from space-borne passive sensors. RTTOV has already been used for many years by many na- tional meteorological services for assimilating downwardlooking observations from visible, infrared, and microwave radiometers, spectrometers, and interferometers (Hocking et al., 2015, and references therein) aboard satellite platforms. The FORTRAN-90 code originally developed at ECMWF in the early 90s (Eyre, 1991) was intended for TOVS direct radiance assimilation within three- and four-dimensional variational analysis schemes (3DVAR, 4DVAR). Subsequently the original code has gone through several developments (e.g., Saunders et al., 1999; Matricardi et al., 2001), more recently within the EUMETSAT NWPSAF, of which RTTOV v11.3 is the latest version available. Since its first implementation and throughout its current version, RTTOV has been developed and exploited for satellite observation perspective only. The model allows rapid simulations of radiance for a suite of passive sensors given the atmospheric state vector, i.e., profiles of temperature, gas concentration, cloud liquid water content, and surface properties. The only one variable gas needed for RTTOV v11 in the microwave band is water vapor. An important feature of RTTOV is that, in addition to the forward (or direct) radiative transfer, it also computes the Jacobians, i.e., the gradient of the radiance with respect to the state vector at the location in state space specified by the input state vector values. The Jacobians are calculated in tangent linear (TL), adjoint (AD), and K-modules of RTTOV.

There are other fast RT models used by the NWP community for satellite data assimilation, like the Community Radiative Transfer Model (CRTM - Ding et al., 2011). However, to our knowledge no fast RT model is currently available to simulate ground-based radiometric observations. In this work, version 11.2 of RTTOV has been modified to handle ground-based microwave radiometer observations. The efforts for adapting RTTOV to ground-based observations started within the COST action ES1202 (EG-CLIMET) and have been continued within the COST action ES1303 (TOPROF). The ground-based version of RTTOV developed here, called RTTOV-gb, is able to simulate brightness temperatures from ground-based upward-looking microwave radiometers. In addition, the TL, AD, and K-modules of RTTOV have been adapted to provide Jacobians for groundbased geometry. We believe that the availability of RTTOV$\mathrm{gb}$ with its K-module will enable more widespread and better use of MWR observations in NWP models.

This paper introduces RTTOV-gb, the ground-based version of the fast radiative transfer model RTTOV. In Sect. 2 we describe the modifications made to the original RTTOV code for the ground-based radiative transfer calculation. Section 3 discusses the performance of RTTOV-gb by evaluating its simulations against those from accurate line-by-line RT models (3.1), against ground-based real MWR observations (3.2), against analytic Jacobian calculations (3.3), and finally within a one-dimensional variational (1D-Var) assimilation scheme (3.4). Section 4 summarizes the findings and draws the final conclusions. 


\section{The formulation of the radiative transfer model}

\subsection{Radiative transfer model}

Given a state vector $\boldsymbol{x}$ (the atmospheric thermodynamical state profile in radiative transfer problem), the radiance vector (or brightness temperature) $\boldsymbol{y}$ is computed as

$\boldsymbol{y}=H(\boldsymbol{x})$,

where $H$ is the radiative transfer model (also referred to as the observation operator).

The core of RTTOV-gb simulates ground-based radiometer radiance using an approximated form of the radiative transfer equation (RTE) for ground-based (upward-looking) observation geometry:

$$
L_{\mathrm{ATM}, i}=\tau_{i, \text { toa }} \cdot B_{i}\left(T_{\mathrm{BKG}}\right)+\int_{\tau_{i, \text { toa }}}^{1} B_{i}(T) \mathrm{d} \tau,
$$

where $L_{\mathrm{ATM}, i}$ is the radiance at the ground for channel $i$, neglecting scattering effects, $B_{i}$ is the Planck radiance at channel $i$ for a scene temperature $T, \tau_{i, \text { toa }}$ is the transmittance from the surface to the top of the atmosphere, and $T_{\mathrm{BKG}}$ is the microwave cosmic background temperature $(2.728 \mathrm{~K})$. Note that in the spectral range under consideration $(20-60 \mathrm{GHz})$, scattering is negligible for particles of the size of atmospheric molecules and cloud droplets, and even for larger ice and snow particles (Kneifel et al., 2010). From a ground-based perspective, the transmittances and optical depths are accumulated from the surface to the space instead of from the space to the surface as in the original RTTOV satellite perspective. Consequently, several subroutines have been modified to reverse the accumulation of transmittances and optical depths through the atmospheric path (see Sect. 6).

The RTE (2) is valid for both clear- and cloudy-sky conditions because in the microwave band, RTTOV takes the liquid water as an absorbing species into account, and its effects are included through a contribution to the transmittance profile. The first term of the right-hand side of the RTE (2) is the cosmic background radiation; the second term is the atmospheric contribution.

The RTE (2) has been numerically solved over $N$ atmospheric levels which are numbered from the top of the atmosphere as follows:

- level $j=1$, pressure $P_{j}=0.005 \mathrm{hPa}$, temperature $T_{j}=$ $T_{1}$, transmittance $\tau_{i j}=\tau_{i, \text { toa }}$ for channel $i$;

- levels from $j=2$ to $j=N-1, P_{j}$ are pressures of the fixed-pressure levels, $\tau_{i j}$ is the surface-to-level transmittance for channel $i$;

- level $j=M$, the first level which lies strictly above the input $2 \mathrm{~m}$ pressure (i.e., $M<=N$ and $P_{M}<P_{2} \mathrm{~m}$ ), $\tau_{i j}=\tau_{i, M}$ for channel $i$;
- level $j=N, P_{N}=1050 \mathrm{hPa}$, surface air temperature $T_{N}=T_{S}, \tau_{i N}=1$ for all channels.

For the ground-based perspective and each channel (omitting the $i$ index for convenience), we define

$\left\{\begin{array}{l}\Delta \tau_{j}=\tau_{j+1}-\tau_{j} \\ \Delta B_{j}=B_{j}-B_{j+1} \\ \Delta d_{j}=d_{j}-d_{j+1}\end{array}\right.$

where $\Delta d_{j}$ is the optical depth of the single layer $j$, and $d_{j}$ is the level-to-surface optical depth.

The contribution of the cosmic background radiation is

$L_{\mathrm{COSMIC}}=\tau_{1} \cdot B\left(T_{\mathrm{BKG}}\right)$ with $\tau_{1}=\tau_{\mathrm{toa}}$.

The atmospheric contribution is

$L_{A}=\int_{\tau_{\mathrm{LEV}=1}}^{\tau_{\mathrm{M}}} B(T) \mathrm{d} \tau+\mathrm{ST}=\sum_{j=M}^{1}\left(\int_{\tau_{j}}^{\tau_{j+1}} B d \tau\right)+\mathrm{ST}$,

where

$$
\begin{aligned}
\int_{\tau_{j}}^{\tau_{j+1}} B d \tau & =\tau_{j+1} B_{j+1}-\tau_{j} B_{j}+\frac{1}{\Delta d_{j}} \Delta B_{j} \Delta \tau_{j} \\
& =\Delta \tau_{j} \cdot\left[B_{j+1}+\Delta B_{j} \frac{1}{\Delta d_{j}}\right]-\tau_{j} \Delta B_{j},
\end{aligned}
$$

and ST is the contribution of the first layer above the surface:

$$
\begin{aligned}
& \mathrm{ST}=B_{S}\left(1-\tau_{M}\right)-\left(B_{M}-B_{S}\right)+\left(B_{M}-B_{S}\right) \\
& \quad \cdot\left(1-\tau_{M}\right) \cdot \frac{1}{d_{M}},
\end{aligned}
$$

with $B_{S}$ the Planck function evaluated at the input $2 \mathrm{~m}$ temperature.

In Eq. (4) we have used a parameterization of the Planck function (i.e., the so-called linear-in-tau assumption, where tau means the optical depth of the single layer, corresponding to $\Delta d$ in the notation used in this study). In the linear-in-tau assumption, the source function throughout the layer is linear with the optical depth of the layer (Saunders, 2010):

$$
B[T(\Delta d)]=B_{j+1}+\left(B_{j}-B_{j+1}\right) \frac{\Delta d}{\Delta d_{j}},
$$

where $B_{j}$ is the Planck function for the top of the layer, $B_{j+1}$ is the Planck function at the bottom of the layer, and $\Delta d_{j}$ is the optical depth of the layer. In the ground-based perspective, $\Delta d$ goes from 0 to $\Delta d_{j}$ from the bottom to the top of the layer.

The radiance for each channel $i$ is then converted to an equivalent black-body temperature, which is usually called brightness temperature (TB), using the inverse Planck function. 


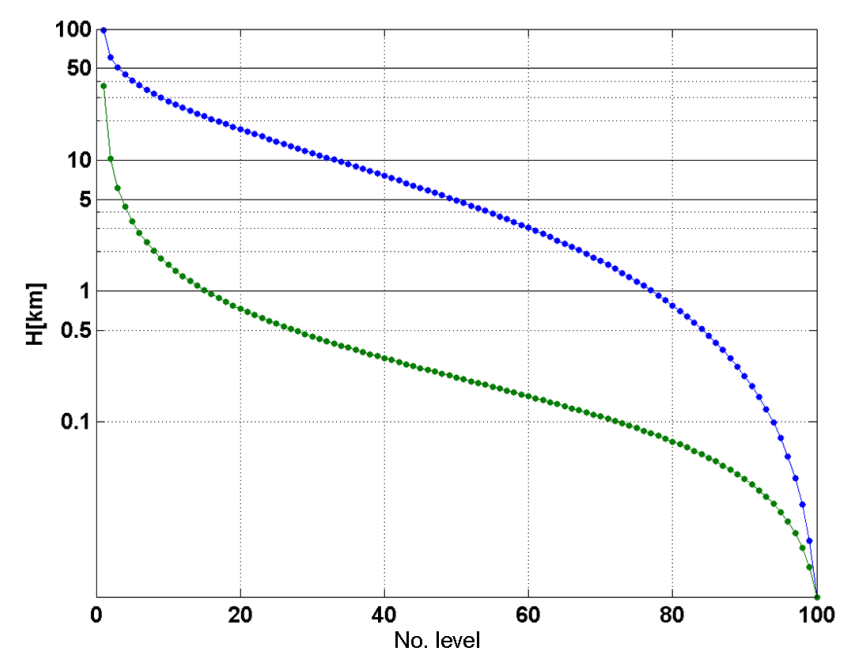

Figure 1. Vertical spacing of profiles levels used for RTTOV in this analysis. Level altitudes and altitude differences between levels are reported respectively with blue and green lines. Note that the $y$ axis is in logarithmic scale.

\subsection{The input atmospheric profiles and near-surface variables}

The input profile data may be supplied on an arbitrary set of pressure levels. These consist of vertical profiles of temperature (K) and humidity (ppmv) for clear-sky conditions, and additional cloud liquid water content (CLW in $\mathrm{kg} \mathrm{kg}^{-1}$ ) profiles for simulating cloudy conditions. In addition, pressure, temperature, and humidity values at $2 \mathrm{~m}$ altitude are required. The transmittance calculations described below are performed using atmospheric layers bounded by a number of fixed pressure levels. RTTOV-gb interpolates the input profiles to the fixed pressure levels for the transmittance calculation, but note that the RTE is integrated in the pressure levels supplied by the user (Hocking, 2014).

Currently RTTOV-gb uses fixed 101 pressure levels from 0.005 to $1050 \mathrm{hPa}$ for the transmittance calculation. These levels have been specifically selected for the ground-based perspective to be denser close to ground (34 levels below $2 \mathrm{~km}$ ) than those usually used for the satellite perspective. Moreover they were chosen to improve the accuracy of the optical depth prediction scheme used by RTTOV-gb compared to that obtained with the levels used for satellite simulations. The vertical spacing of levels is shown in Fig. 1 in terms of level altitude differences.

\subsection{Transmittance model}

The main variable computed in the radiative transfer model is the atmospheric optical depth for each channel $i$ and for each atmospheric layer $j$. The optical depths depend on the viewing angle of the instrument, pressure, temperature, and concentrations of the absorbing species. The optical depth differ- ences between adjacent pressure levels are obtained through a linear combination in $X_{k j}$, the so-called predictors ( $j$ being the level and $k$ the number of predictors, from 1 to $N_{P}$ ). The predictors are derived from the input state vector profile and depend on the elevation angle $\theta$ and pressure $P$, temperature $T$, and specific humidity $\mathrm{q}$ at the considered level and the level above that. The optical depth from the surface to the level $j$ in channel $i$ along a path at an angle $\theta$ from the vertical, $d_{i j}$, is obtained as follows:

$d_{i j}=d_{i, j+1}+\sum_{k=1}^{P} a_{i j k} X_{k j}(P, T, q, \theta)$,

with $a_{i j k}$ the regression coefficients between optical depths and predictors.

The contribution of the water vapor to the optical depth is treated separately from that of uniformly mixed gases although they are calculated with two algorithms of the same form. There are three types of predictors for satellite perspective, predictors 7 (Matricardi et al., 2001), 8 (Matricardi, 2005), and 9 (Saunders, 2010), each of which is better suited for a specific application. The predictors used in RTTOV to parameterize the optical depths refer to the reference temperature and specific humidity profiles (i.e., the average of the training profile set, respectively $T_{j}^{\text {ref }}$ and $q_{j}^{\text {ref }}$ ). Additionally, the number of predictors depends on the selected gas.

We found the predictors 7 to give the best results for the ground-based geometry, and thus they are used herewith to train RTTOV-gb. The predictors 7 and the profile variables involved in the predictors calculation are listed in Appendix A. Note that predictors 7 were originally developed for satellite simulations up to $60^{\circ}$ zenith angles and as such, the errors in the optical depth prediction increase for zenith angles above $\sim 75^{\circ}$ (i.e., for elevation angles below $\sim 15^{\circ}$ ). For MWR observations of the PBL thermodynamics, these scanning angles turn out to be crucial because of the information carried by opaque channels on the PBL temperature profile. Thus, it is foreseen that an alternative set of predictors, specific for low-elevation angles in the ground-based geometry, may be worth investigating and developing in the future, though it is beyond the scope of this study.

The coefficients $a_{i k j}$ are calculated by linear regression of $\left\{d_{i, j}-d_{i, j+1}\right\}$ against $X_{k j}$. For the regression, $d_{i j}$ are calculated using a line-by-line (LBL) model for a set of atmospheric profiles. LBL RT models provide accurate calculation of the atmospheric transmittances and radiance, given the atmospheric profile of gas concentrations, and predefined spectral frequency grid. The LBL optical depths must cover the full spectral range of all the radiometer channels of interest and provide a sufficient resolution to represent the transmittances in the channel spectral bands accurately. The LBL model described by Rosenkranz (1998; R98 herewith) has been used for gas absorption to calculate the clear-sky transmittances needed in the RTTOV-gb regression coefficients' computation. Here we use 83 profiles from an NW- 
PSAF profile dataset interpolated on 101 pressure levels, already used for training RTTOV. It is important to emphasize that this profile set was carefully chosen from a set of more than 100 million profiles to represent a wide range of physically realistic atmospheric states (Matricardi, 2008). Transmittances are computed for six selected scanning angles which are discussed in Sect. 3.1. We limit the lowest elevation angle used in the training phase to $10^{\circ}$ because of the limitation of the predictors 7 that has already been mentioned.

If the optical depths for uniformly mixed gases and water vapor are $d_{i j}^{M}$ and $d_{i j}^{W}$ respectively, the total optical depth is

$d_{i j}=d_{i j}^{M}+d_{i j}^{W}$.

Then, optical depths are converted to transmittances:

$\tau_{i j}=\exp \left(-d_{i j}\right)$

Finally, RTTOV-gb computes the output radiance and TB from the derived transmittances and the input vertical temperature profile using the radiative transfer Eq. (2).

\subsection{Jacobians: tangent linear, adjoint, and gradient matrix models}

The Jacobian matrix $\mathbf{K}$ gives the change in radiance $\delta \boldsymbol{y}$ for a change in any element of the state vector $\delta \boldsymbol{x}$, assuming a linear relationship about a given atmospheric state $\boldsymbol{x}_{0}$ :

$\delta y=\mathbf{K}\left(x_{0}\right) \delta x$.

The elements of $\mathbf{K}$ contain the partial derivatives $\delta y_{i} / \delta x_{j}$, where the subscript $i$ refers to the channel and $j$ to the layer number. The Jacobian provides the radiance sensitivity for each channel given unit perturbations at each level of the state vector and in each of the surface parameters. It shows clearly, for a given profile, which layers in the atmosphere are most sensitive to changes in temperature and variable gas concentrations for each channel. The K-module of RTTOV computes the $\mathbf{K}\left(\boldsymbol{x}_{0}\right)$ matrix for each input profile. Alternatively, the Jacobians can be computed with the so-called brute force (BF) method, where $\mathbf{K}$ is estimated by perturbing each element of the atmospheric state vector, repeating the RTTOV direct module iteratively. However, the calculations of the Jacobians with the BF method are slower and less rigorous than with the K-module of RTTOV.

It is not always necessary to store and access the full matrix $\mathbf{K}$; thus, the RTTOV package has routines to compute the tangent linear only, i.e., the change in radiance $y_{i}$ for a given change in atmospheric profile $\boldsymbol{\delta} \boldsymbol{x}$ around an initial atmospheric state $\boldsymbol{x}_{0}$

$$
\begin{gathered}
\delta y\left(x_{0}\right)=\left[\delta x \frac{\partial y_{1}}{\partial x}, \delta x \frac{\partial y_{2}}{\partial x}, \delta x \frac{\partial y_{3}}{\partial x} \ldots \delta x \frac{\partial y_{\text {nchan }}}{\partial x}\right] \\
\text { with } \frac{\partial}{\partial x}=\nabla_{x}=\left[\frac{\partial}{\partial x_{1}}, \frac{\partial}{\partial x_{2}}, \ldots, \frac{\partial}{\partial x_{N}}\right]
\end{gathered}
$$

Similarly, the adjoint routines compute the change in any quantity of the state vector (e.g., $T, q$, surface variables etc.) $\boldsymbol{\delta} \boldsymbol{x}$ around an assumed atmospheric state $\boldsymbol{x}_{0}$, given a change in the radiance $\boldsymbol{\delta} \boldsymbol{y}$.

$$
\begin{aligned}
& \nabla_{x}=\nabla_{x} y \cdot \nabla_{y}=K\left(x_{0}\right)^{\mathrm{T}} \cdot \nabla_{y} \\
& \delta \boldsymbol{x}\left(\boldsymbol{x}_{0}\right)=\left[\delta \boldsymbol{y} \frac{\partial x_{1}}{\partial \boldsymbol{y}}, \delta y \frac{\partial x_{2}}{\partial \boldsymbol{y}}, \delta y \frac{\partial x_{3}}{\partial y} \ldots \delta y \frac{\partial x_{N}}{\partial \boldsymbol{y}}\right]
\end{aligned}
$$

For very large systems, it may be not feasible to calculate the full Jacobian matrix $\mathbf{K}$, and the tangent linear and adjoint operations are computed instead.

The TL code is derived directly from the forward model because it represents the analytic derivative of the radiance (forward model outputs) with respect to the atmospheric state vector $\boldsymbol{x}$. The AD code is derived from the TL code. Finally, the $\mathrm{K}$ code is obtained from the $\mathrm{AD}$ code distributing the $\mathrm{AD}$ level derivatives through the number of channels. Before running $\mathrm{TL}, \mathrm{AD}$, and $\mathrm{K}$ models, the direct model needs to be run because many of the intermediate variables calculated by the direct model are needed by the TL, AD, and K-modules.

\section{Performance of RTTOV-gb}

The performance of RTTOV-gb has been tested in four different ways, reported in the following subsections: validation against the LBL RT model used as reference for the training and against another independent reference LBL RT model (3.1); a comparison of TB simulated with RTTOV-gb from a radiosonde profile dataset with nearly co-located MWR measurements (3.2); a comparison of Jacobians calculated with the RTTOV-gb K-module and the brute force method, and also with Jacobians computed with an analytical model (3.3); the exploitation of RTTOV-gb as a forward model operator within a one-dimensional variational scheme (3.4).

\subsection{Comparison with line-by-line model computed radiance}

To compare RTTOV-gb against the LBL model adopted for the regression training, we computed clear-sky TB with both RTTOV-gb and R98 at selected channels from the set of 83 atmospheric profiles used in the training phase. Resulting $\mathrm{TB}$ differences are a measure of the regression error. Here we focus on the systematic (bias) and root-mean-square (rms) TB differences. We consider 14 channels commonly used by commercial MWRs, in particular the Humidity And Temperature PROfiler (HATPRO, Rose et al., 2005): 22.24, 23.04, 23.84, 25.44, 26.24, 27.84, 31.40, 51.26, 52.28, 53.86, $54.94,56.66,57.30$, and $58.00 \mathrm{GHz}$. Channels from 22 to $31 \mathrm{GHz}$ are in the so-called K-band, while channels from 51 to $58 \mathrm{GHz}$ are in the so-called V-band.

Table 1 shows bias and rms at four elevation angles (90, 30,19 , and $10^{\circ}$ ) and four elevation angle combinations used 
Table 1. Statistics for the comparison between RTTOV-gb and the line-by-line model R98 (Rosenkranz, 1998) at elevation angles 90, 30, 19, and $10^{\circ}$ (R98 minus RTTOV-gb). The HATPRO channel number (Chan no.), the channel central frequency, bias, and rms for each RTTOV training configuration are reported. The values which are larger than $0.5 \mathrm{~K}$ are highlighted in bold.

\begin{tabular}{|c|c|c|c|c|c|c|c|c|c|}
\hline \multirow[b]{3}{*}{ Chan no. } & \multirow[b]{3}{*}{ Frequency $(\mathrm{GHz})$} & \multicolumn{4}{|c|}{$\operatorname{Bias}(\mathrm{K})$} & \multicolumn{4}{|c|}{$\operatorname{rms}(\mathrm{K})$} \\
\hline & & \multicolumn{8}{|c|}{ Elevation angle $90^{\circ}$} \\
\hline & & $90-26^{\circ}$ & $90-16^{\circ}$ & $90-10^{\circ}$ & $90-5^{\circ}$ & $90-26^{\circ}$ & $90-16^{\circ}$ & $90-10^{\circ}$ & $90-5^{\circ}$ \\
\hline 1 & 22.24 & -0.007 & -0.016 & 0.004 & -0.170 & 0.033 & 0.059 & 0.047 & 0.373 \\
\hline 2 & 23.04 & -0.002 & -0.009 & 0.011 & -0.159 & 0.029 & 0.052 & 0.050 & 0.352 \\
\hline 3 & 23.84 & 0.005 & 0.002 & 0.023 & -0.132 & 0.028 & 0.043 & 0.053 & 0.308 \\
\hline 4 & 25.44 & 0.009 & 0.011 & 0.029 & -0.087 & 0.029 & 0.036 & 0.056 & 0.224 \\
\hline 5 & 26.24 & 0.009 & 0.011 & 0.028 & -0.074 & 0.029 & 0.035 & 0.054 & 0.195 \\
\hline 6 & 27.84 & 0.008 & 0.012 & 0.025 & -0.059 & 0.029 & 0.034 & 0.050 & 0.158 \\
\hline 7 & 31.40 & 0.009 & 0.011 & 0.023 & -0.049 & 0.033 & 0.038 & 0.049 & 0.128 \\
\hline 8 & 51.26 & 0.017 & 0.024 & 0.043 & -0.101 & 0.175 & 0.176 & 0.159 & 0.244 \\
\hline 9 & 52.28 & 0.021 & 0.025 & 0.039 & -0.070 & 0.202 & 0.201 & 0.186 & 0.246 \\
\hline 10 & 53.86 & 0.010 & 0.012 & 0.015 & 0.001 & 0.116 & 0.118 & 0.115 & 0.122 \\
\hline 11 & 54.94 & 0.002 & 0.003 & 0.004 & -0.008 & 0.023 & 0.023 & 0.023 & 0.023 \\
\hline 12 & 56.66 & 0.001 & 0.001 & 0.001 & 0.001 & 0.007 & 0.007 & 0.007 & 0.007 \\
\hline 13 & 57.30 & 0.001 & 0.001 & 0.001 & 0.001 & 0.005 & 0.005 & 0.005 & 0.005 \\
\hline \multirow[t]{2}{*}{14} & 58.00 & 0.000 & 0.001 & 0.001 & 0.001 & 0.004 & 0.004 & 0.004 & 0.004 \\
\hline & & \multicolumn{8}{|c|}{ Elevation angle $30^{\circ}$} \\
\hline Chan no. & Frequency $(\mathrm{GHz})$ & $90-26^{\circ}$ & $90-16^{\circ}$ & $90-10^{\circ}$ & $90-5^{\circ}$ & $90-26^{\circ}$ & $90-16^{\circ}$ & $90-10^{\circ}$ & $90-5^{\circ}$ \\
\hline 1 & 22.24 & 0.002 & 0.027 & 0.020 & 0.036 & 0.033 & 0.047 & 0.046 & 0.180 \\
\hline 2 & 23.04 & 0.000 & 0.025 & 0.019 & 0.029 & 0.030 & 0.047 & 0.043 & 0.173 \\
\hline 3 & 23.84 & -0.002 & 0.020 & 0.016 & 0.014 & 0.026 & 0.040 & 0.040 & 0.162 \\
\hline 4 & 25.44 & -0.004 & 0.013 & 0.013 & -0.007 & 0.024 & 0.037 & 0.037 & 0.150 \\
\hline 5 & 26.24 & -0.004 & 0.010 & 0.012 & -0.012 & 0.023 & 0.036 & 0.035 & 0.145 \\
\hline 6 & 27.84 & -0.003 & 0.008 & 0.011 & -0.016 & 0.024 & 0.037 & 0.033 & 0.137 \\
\hline 7 & 31.40 & -0.004 & 0.006 & 0.010 & -0.019 & 0.155 & 0.043 & 0.036 & 0.131 \\
\hline 8 & 51.26 & 0.010 & 0.018 & 0.027 & -0.079 & 0.029 & 0.171 & 0.162 & 0.211 \\
\hline 9 & 52.28 & 0.016 & 0.019 & 0.026 & -0.073 & 0.138 & 0.149 & 0.143 & 0.174 \\
\hline 10 & 53.86 & 0.003 & 0.007 & 0.008 & -0.005 & 0.026 & 0.028 & 0.028 & 0.027 \\
\hline 11 & 54.94 & 0.001 & 0.001 & 0.001 & -0.001 & 0.007 & 0.007 & 0.007 & 0.007 \\
\hline 12 & 56.66 & 0.000 & 0.000 & 0.000 & -0.000 & 0.002 & 0.002 & 0.002 & 0.002 \\
\hline 13 & 57.30 & 0.000 & 0.000 & 0.000 & -0.000 & 0.001 & 0.001 & 0.001 & 0.001 \\
\hline 14 & 58.00 & 0.000 & 0.000 & 0.000 & -0.000 & 0.001 & 0.001 & 0.001 & 0.001 \\
\hline
\end{tabular}

Elevation angle $19^{\circ}$

\begin{tabular}{lrrrrr|rrrr}
\hline Chan no. & Frequency $(\mathrm{GHz})$ & $90-26^{\circ}$ & $90-16^{\circ}$ & \multicolumn{1}{c}{$90-10^{\circ}$} & $90-5^{\circ}$ & $90-26^{\circ}$ & $90-16^{\circ}$ & $90-10^{\circ}$ & $90-5^{\circ}$ \\
\hline 1 & 22.24 & -0.050 & -0.004 & -0.065 & 0.203 & 0.078 & 0.044 & 0.086 & 0.317 \\
2 & 23.04 & -0.053 & -0.005 & -0.070 & 0.189 & 0.079 & 0.042 & 0.089 & 0.298 \\
3 & 23.84 & -0.056 & -0.007 & -0.074 & 0.158 & 0.083 & 0.038 & 0.090 & 0.259 \\
4 & 25.44 & -0.046 & -0.007 & -0.070 & 0.099 & 0.089 & 0.036 & 0.087 & 0.192 \\
5 & 26.24 & -0.039 & -0.006 & -0.066 & 0.080 & 0.089 & 0.036 & 0.084 & 0.171 \\
6 & 27.84 & -0.028 & -0.005 & -0.059 & 0.055 & 0.091 & 0.036 & 0.078 & 0.149 \\
7 & 31.40 & -0.018 & -0.004 & -0.052 & 0.035 & 0.103 & 0.043 & 0.077 & 0.139 \\
8 & 51.26 & 0.020 & 0.013 & -0.018 & -0.003 & 0.139 & 0.128 & 0.132 & 0.152 \\
9 & 52.28 & -0.031 & 0.012 & -0.004 & 0.021 & 0.085 & 0.085 & 0.085 & 0.097 \\
10 & 53.86 & 0.004 & 0.001 & -0.000 & 0.003 & 0.013 & 0.010 & 0.010 & 0.011 \\
11 & 54.94 & 0.002 & 0.000 & 0.000 & 0.001 & 0.005 & 0.003 & 0.003 & 0.004 \\
12 & 56.66 & 0.000 & 0.000 & 0.000 & 0.000 & 0.001 & 0.001 & 0.001 & 0.001 \\
13 & 57.30 & 0.000 & 0.000 & 0.000 & 0.000 & 0.000 & 0.000 & 0.000 & 0.000 \\
14 & 58.00 & 0.000 & 0.000 & 0.000 & 0.000 & 0.000 & 0.000 & 0.000 & 0.000 \\
\hline
\end{tabular}


Table 1. Continued.

\begin{tabular}{|c|c|c|c|c|c|c|c|c|c|}
\hline \multirow[b]{3}{*}{ Chan no. } & \multirow[b]{3}{*}{ Frequency $(\mathrm{GHz})$} & \multicolumn{4}{|c|}{ Bias $(\mathrm{K})$} & \multicolumn{4}{|c|}{ rms $(\mathrm{K})$} \\
\hline & & \multicolumn{8}{|c|}{ Elevation angle $10^{\circ}$} \\
\hline & & $90-26^{\circ}$ & $90-16^{\circ}$ & $90-10^{\circ}$ & $90-5^{\circ}$ & $90-26^{\circ}$ & $90-16^{\circ}$ & $90-10^{\circ}$ & $90-5^{\circ}$ \\
\hline 1 & 22.24 & -0.299 & -0.324 & -0.626 & $-\mathbf{0 . 9 3 0}$ & 0.428 & 0.381 & 0.681 & 1.035 \\
\hline 2 & 23.04 & -0.297 & -0.317 & -0.632 & -0.955 & 0.461 & 0.369 & 0.685 & 1.027 \\
\hline 3 & 23.84 & -0.391 & -0.312 & -0.648 & -0.998 & 0.662 & 0.356 & 0.698 & 1.067 \\
\hline 4 & 25.44 & -0.544 & -0.294 & -0.664 & -1.055 & 1.214 & 0.343 & 0.716 & 1.128 \\
\hline 5 & 26.24 & -0.573 & -0.284 & -0.663 & -1.065 & 1.414 & 0.342 & 0.718 & 1.143 \\
\hline 6 & 27.84 & -0.592 & -0.270 & -0.659 & -1.075 & 1.685 & 0.349 & 0.716 & 1.159 \\
\hline 7 & 31.40 & -0.594 & -0.260 & -0.680 & -1.129 & 2.023 & 0.377 & 0.731 & 1.205 \\
\hline 8 & 51.26 & 0.000 & -0.088 & -0.337 & -0.609 & 0.272 & 0.103 & 0.350 & 0.633 \\
\hline 9 & 52.28 & -0.021 & -0.029 & -0.106 & -0.202 & 0.083 & 0.034 & 0.112 & 0.214 \\
\hline 10 & 53.86 & 0.022 & 0.000 & -0.007 & -0.014 & 0.037 & 0.003 & 0.011 & 0.021 \\
\hline 11 & 54.94 & 0.005 & 0.000 & -0.002 & -0.004 & 0.009 & 0.001 & 0.003 & 0.006 \\
\hline 12 & 56.66 & 0.000 & 0.000 & -0.000 & -0.000 & 0.001 & 0.000 & 0.000 & 0.001 \\
\hline 13 & 57.30 & 0.000 & 0.000 & -0.000 & -0.000 & 0.000 & 0.000 & 0.000 & 0.001 \\
\hline 14 & 58.00 & 0.000 & 0.000 & -0.000 & -0.000 & 0.000 & 0.000 & 0.000 & 0.000 \\
\hline
\end{tabular}
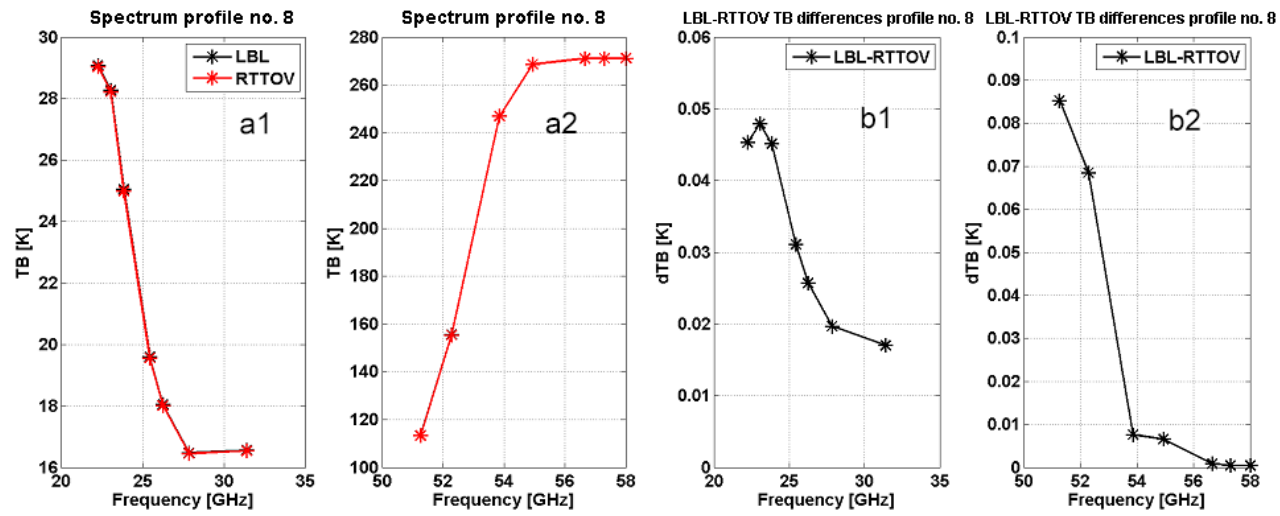

Figure 2. (a1) TB at K-band channels (20-35 GHz) computed by RTTOV-gb (red stars) and LBL R98 (black stars) from profile no. 8 of the dependent set. (a2) Same as (a1), but for V-band channels (50-60 GHz). (b1) TB differences (R98 minus RTTOV-gb) at K-band channels. (b2) Same as (b1), but for V-band channels.

for regression training (predictors 7 are used). This comparison allow us to investigate the best performing combination. The four sets of elevation angles are 90-53-42-35-30-26 90-42-30-24-19-16 ; 90-42-30-24-19-10 ${ }^{\circ}$; and 90-4230-19-10-5 .

Bias and rms are lower than the manufacturer error specification for HATPRO channels ( $\sim 0.5 \mathrm{~K}-$ Rose et al., 2005) for all the considered training sets and elevation angles, with the exception of $22-31 \mathrm{GHz}$ channels at $10^{\circ}$ elevation angle with the training sets $90-26,90-10$, and $90-5^{\circ}$. This result seems to confirm that predictors 7 are not ideal for elevation angles lower than $15^{\circ}$. However, it is encouraging to note that even at $10^{\circ}$, bias and rms are within the instrumental error for all the channels when the training set $90-16^{\circ}$ is adopted. Note that the agreement at low-elevation angles is better for the V-band opaque channels, which are most important for
PBL temperature retrieval. Table 1 shows that the best among the considered training configurations is the set of elevation angles from 90 to $16^{\circ}$. Somewhat surprising, this configuration gives acceptable results even at $10^{\circ}$, despite this elevation angle being outside the training angle range.

Figure 2 shows two spectra computed at HATPRO channels by RTTOV-gb and LBL R98 for the same atmospheric profile belonging to the dependent set. For this particular case, TB differences between the two models are within $0.1 \mathrm{~K}$ for all channels.

For the whole 83-profile dataset, Fig. 3 shows statistics (bias, rms, and maximum value) of the LBL R98 minus RTTOV-gb TB difference at four elevation angles $(90,30,19$, and $10^{\circ}$ ). The best training configuration determined above (elevation angles from 90 to $16^{\circ}$ ) is used. At $90^{\circ}$ elevation, bias and rms are respectively less than 0.030 and $0.060 \mathrm{~K}$ for 

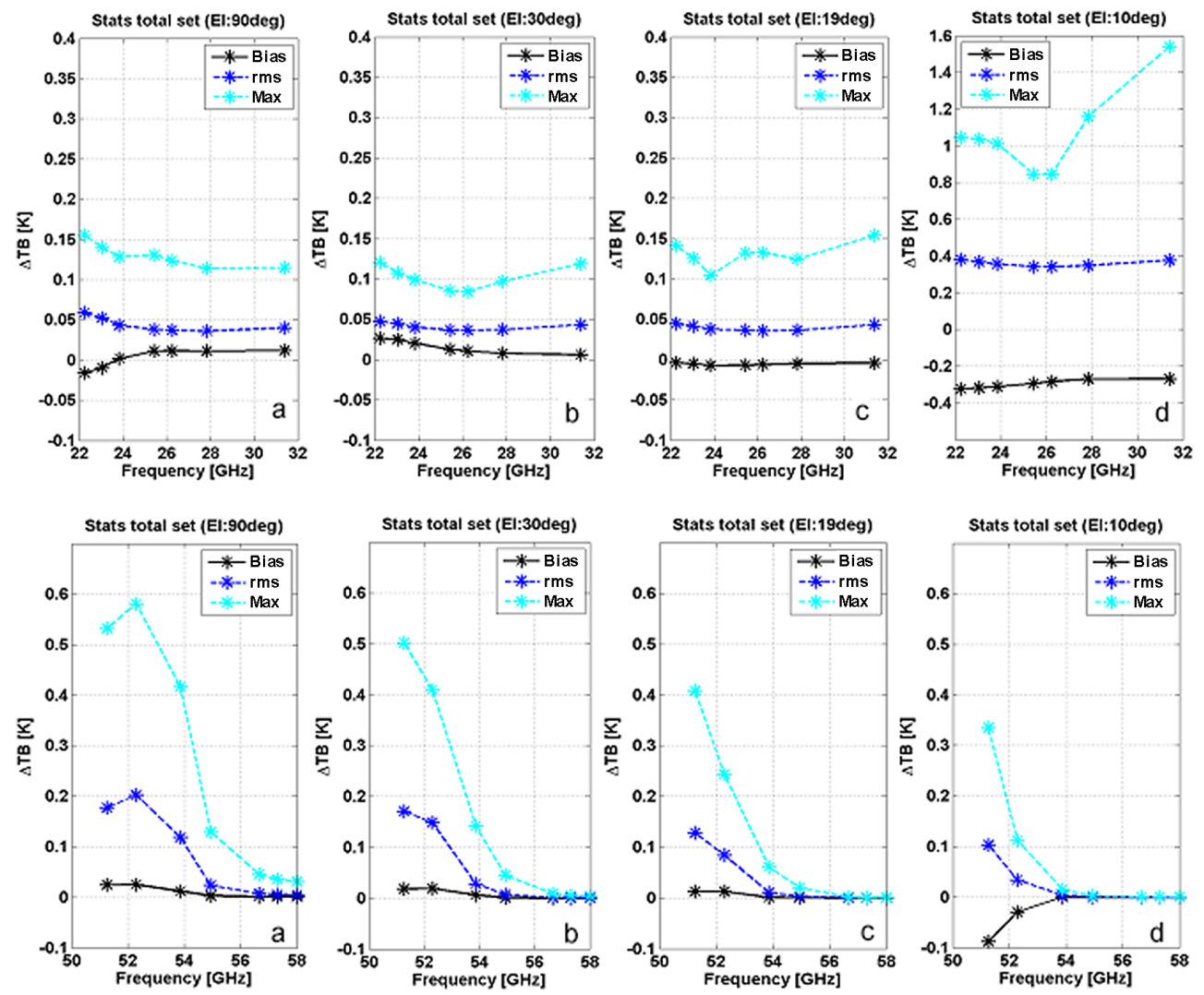

Figure 3. Bias (black solid line), rms (blue dashed line), and maximum (cyan dashed line) of TB difference between RTTOV-gb and LBL R98 (Rosenkranz, 1998) for the dependent 83-profile set and the best training configuration (R98 minus RTTOV-gb). Top panels: K-band channels; bottom panels: V-band channels. Panels (a),(b),(c), and (d) report results at 90, 30, 19, and $10^{\circ}$ elevation angle, respectively. Note that the top panel (d) has a different $y$ axis scale with respect to the other top panels.

K-band $(22-31 \mathrm{GHz})$, while they are 0.003 and $0.025 \mathrm{~K}$ for the V-band opaque channels $(54-58 \mathrm{GHz})$. For these channels the maximum difference does not exceed $0.15 \mathrm{~K}$. The agreement is slightly worse at transparent V-band channels (51-54 GHz), with bias, rms, and maximum difference respectively within $0.03,0.2$, and $0.6 \mathrm{~K}$. The larger discrepancies at transparent V-band channels are probably due to the combined influence of temperature and water vapor, which likely decreases the correlation of layer opacity with the two thermodynamical variables. Similar results are found for other elevation angles, such as 30 and $19^{\circ}$. Note that the error statistics at $90^{\circ}$ elevation (i.e., zenith) are about 1 order of magnitude larger than the analogous statistics of the original nadir-looking RTTOV (Saunders, 2002, 2010). We believe the reason is the behavior of the two terms contributing to the total radiance (Eq. 2), i.e., the background and the atmospheric contributions. Uncertainty in atmospheric optical depth, as those induced by regression, will influence the total radiance through the effects on these two terms. For the satellite (downward-looking) case, these effects tend to compensate due to a warmer background (e.g., overestimated optical depths cause more emission from the atmosphere but less contribution from the relative warmer background). Conversely, for the ground-based perspective there is no compensation of the two terms because of the cold cosmic background (e.g., overestimated optical depths causes more emission from the atmosphere and less contribution from the relative colder background).

Figure 3 shows bias, rms, and maximum difference respectively up to $-0.3,0.4$, and $1.5 \mathrm{~K}$ for K-band channels at $10^{\circ}$ elevation. These are significantly larger compared to higher elevation angles. This is attributed to the use of predictors 7 , which are not designed for elevation angles lower than $15^{\circ}$. This may also be due to the fact that $10^{\circ}$ is outside the elevation angle range used in the training configuration $\left(90-16^{\circ}\right)$. However, Table 1 shows that extending the range of training elevation angles to $10^{\circ}$ or less generally degrades statistics. In any case, we highlight that the rms errors in Fig. 3 are smaller than the uncertainty associated with TB observations $(\sim 0.5 \mathrm{~K})$ for all channels and all elevation angles.

Similarly, RTTOV-gb has been compared with the reference LBL model using an independent set of 52 profiles (i.e., not used for training). The same 101 pressure levels described earlier are used. Bias, rms, and maximum of LBL 
Table 2. Statistics for the comparison between RTTOV-gb and the line-by-line model R98 (Rosenkranz, 1998) with the best RTTOV training configuration and the independent profile set (R98 minus RTTOV-gb). HATPRO channel number (Chan no.), the channel central frequency, bias, and rms at elevation angles $90,30,19$, and $10^{\circ}$ are reported.

\begin{tabular}{lrrrrr|rrrr}
\hline & & \multicolumn{5}{c}{ Training configuration: elevation angles from 90 to $16^{\circ}$} \\
\hline & & \multicolumn{3}{c}{ Bias $(\mathrm{K})$} & \multicolumn{4}{c}{ rms (K) } \\
\hline Chan no. & Frequency $(\mathrm{GHz})$ & $90^{\circ}$ & $30^{\circ}$ & $19^{\circ}$ & $10^{\circ}$ & $90^{\circ}$ & $30^{\circ}$ & $19^{\circ}$ & $10^{\circ}$ \\
\hline 1 & 22.24 & -0.008 & 0.021 & -0.004 & -0.282 & 0.049 & 0.045 & 0.042 & 0.326 \\
2 & 23.04 & -0.002 & 0.020 & -0.006 & -0.276 & 0.042 & 0.045 & 0.042 & 0.319 \\
3 & 23.84 & 0.007 & 0.017 & -0.008 & -0.273 & 0.035 & 0.044 & 0.045 & 0.320 \\
4 & 25.44 & 0.018 & 0.001 & -0.009 & -0.257 & 0.032 & 0.042 & 0.051 & 0.339 \\
5 & 26.24 & 0.011 & 0.007 & -0.009 & -0.247 & 0.031 & 0.041 & 0.052 & 0.342 \\
6 & 27.84 & 0.009 & 0.004 & -0.008 & -0.232 & 0.031 & 0.040 & 0.053 & 0.346 \\
7 & 31.40 & 0.008 & 0.001 & -0.010 & -0.230 & 0.036 & 0.046 & 0.061 & 0.365 \\
8 & 51.26 & -0.004 & -0.017 & -0.015 & -0.094 & 0.156 & 0.159 & 0.127 & 0.115 \\
9 & 52.28 & -0.004 & -0.009 & -0.004 & -0.033 & 0.169 & 0.131 & 0.076 & 0.039 \\
10 & 53.86 & -0.001 & 0.002 & -0.001 & -0.002 & 0.095 & 0.025 & 0.015 & 0.012 \\
11 & 54.94 & 0.002 & 0.000 & -0.000 & -0.000 & 0.023 & 0.011 & 0.008 & 0.003 \\
12 & 56.66 & 0.002 & 0.000 & 0.000 & 0.000 & 0.010 & 0.004 & 0.002 & 0.000 \\
13 & 57.30 & 0.001 & 0.000 & 0.000 & 0.000 & 0.009 & 0.003 & 0.001 & 0.000 \\
14 & 58.00 & 0.001 & 0.000 & 0.000 & 0.000 & 0.008 & 0.002 & 0.001 & 0.000 \\
\hline
\end{tabular}

R98 minus RTTOV-gb TB differences are shown in Fig. 4. Results are for the best training configuration and for elevation angles $90,30,19$, and $10^{\circ}$. Statistics are similar to those obtained with the dependent profile set. In this case, however, the error statistics are of the same order of magnitude as the analogous performance of the original nadir-looking RTTOV with an independent profile set (Saunders, 2002, 2010). For elevation angles down to $19^{\circ}$, biases range from less than $0.002 \mathrm{~K}$ for the opaque channels to $0.020 \mathrm{~K}$ for K-band, while rms is less than $0.060 \mathrm{~K}$ for K-band and $0.025 \mathrm{~K}$ for the opaque channels. The maximum TB differences do not exceed $0.5 \mathrm{~K}$. Similarly to the test with the dependent profile set, larger discrepancies are found in the transparent V-band channels (51-54 GHz) and for K-band channels at $10^{\circ}$ elevation. All the statistics obtained with the independent profile set and the best training configuration are summarized in Table 2 . Consistently with the dependent test, the independent test in Fig. 4 and Table 2 confirms that the rms errors are smaller than the uncertainty associated with $\mathrm{TB}$ observations for all channels and all elevation angles.

The previous tests against the reference LBL R98 model have also been performed at the 22 frequency channels (22$60 \mathrm{GHz}$ ) used by another commercial microwave radiometer, the MP-3000A (Cimini et al., 2011, 2015). Statistics, reported in Table 3 in terms of bias and rms, are similar to those obtained for HATPRO channels, at both K- and V-band.

Note that LBL R98 is the model used to train the regression scheme. In order to perform a completely independent test, we compare RTTOV-gb with an independent reference radiative transfer model, the Atmospheric Radiative Transfer Simulator (ARTS, Buehler et al., 2005; Eriks- son et al., 2011; Eriksson and Buehler, 2015), and a completely different profile dataset. In this test, HATPRO observations are simulated using RTTOV-gb and ARTS from a set of 1327 thermodynamic profiles from the AROME analysis over Bordeaux from April to October 2014. AROME is the French convective-scale NWP model with a $2.5 \mathrm{~km}$ horizontal grid mesh developed by Météo France (Seity et al., 2010). Both clear- and cloudy-sky conditions are considered. This dataset, which is limited in space, time, and thus in atmospheric conditions, was chosen to demonstrate the performance of RTTOV-gb in typical deployment environment. Since the goal of this analysis is to test the fast RT modeling (RTTOV-gb) with respect to accurate LBL calculation, all other settings being equal, ARTS settings for absorption model have been selected to adopt the same absorption model as RTTOV-gb as much as possible: R98 for oxygen and water vapor absorption, and the model described in Liebe et al. (1993) for cloud liquid water (referred as MPM93 within ARTS). Note that MPM93 is the only option for liquid water absorption available in ARTS. Conversely RTTOV-gb is consistent with the original RTTOV, which adopts a combination of Liebe et al. (1991) and Lamkaouchy et al. (1997) models (English et al., 1999).

This comparison is presented in Fig. 5 in terms of bias, standard deviation (SD), and rms of ARTS minus RTTOV$\mathrm{gb} \mathrm{TB}$ differences at $90^{\circ}$ elevation angle. Here we have discarded TB differences that are larger than $3 \mathrm{SD}$ from the mean (21 profiles out of 1327 ). Biases less than $0.18 \mathrm{~K}$ for $\mathrm{K}$-band and less than $0.08 \mathrm{~K}$ for opaque V-band channels are found. The rms and standard deviation are close, ranging from 0.1 to $0.25 \mathrm{~K}$ for K-band channels, and within $0.1 \mathrm{~K}$ 

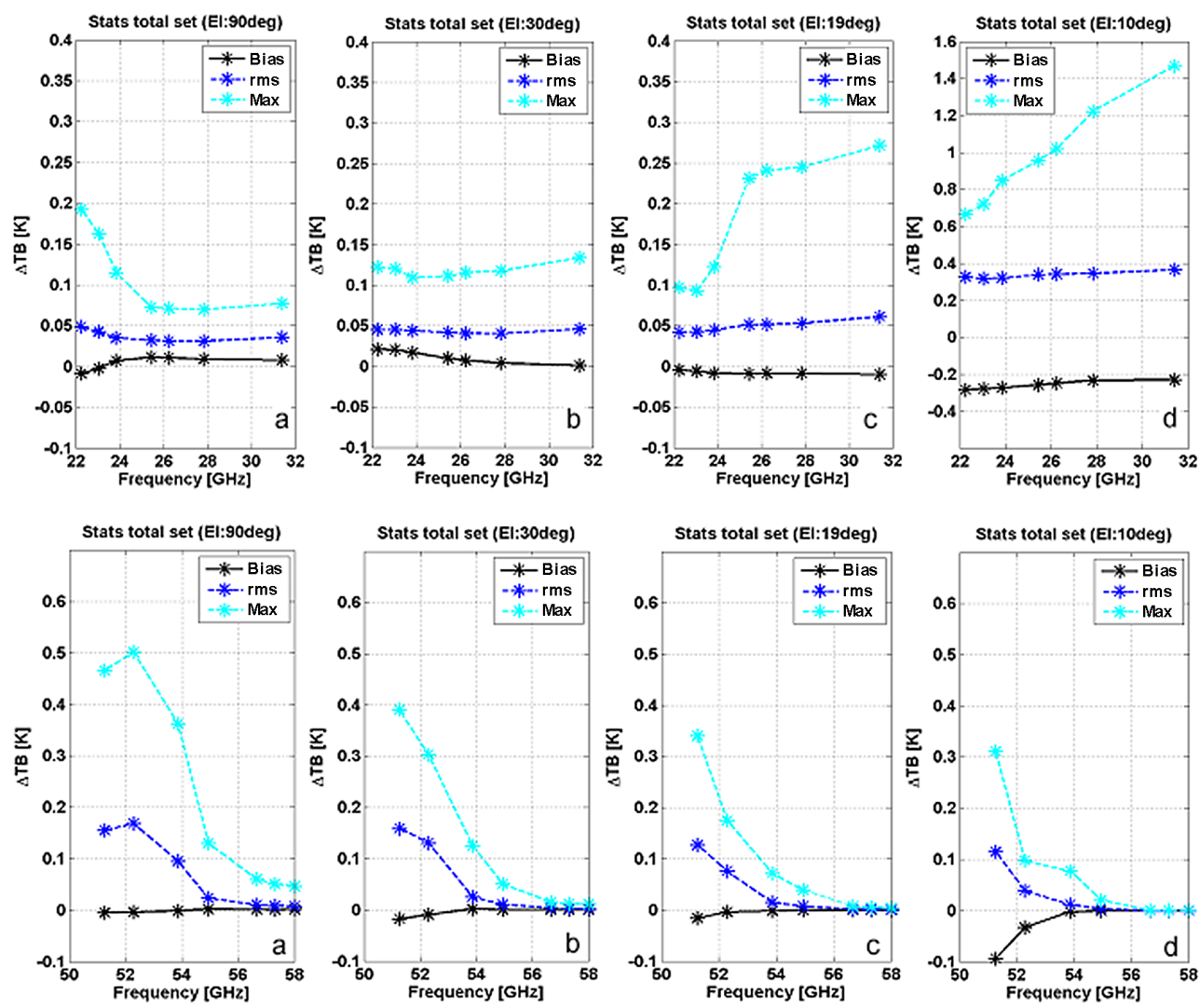

Figure 4. Same as Fig. 3 but for the independent 52-profile set (R98 minus RTTOV-gb). Top panels: K-band channels; bottom panels: V-band channels. Panels (a),(b),(c), and (d) report results at 90, 30, 19, and $10^{\circ}$ elevation angle, respectively. Note that top panel (d) has a different $y$ axis scale to the other top panels.

for V-band opaque channels (55-58 GHz). Similar to previous tests, larger discrepancies are found in the more transparent V-band channels $(51-54 \mathrm{GHz})$ with an rms error up to $0.5 \mathrm{~K}$ at $51 \mathrm{GHz}$ in cloudy-sky; but here the rms is dominated by a bias contribution induced by systematic differences found between LBL and ARTS at these three channels ( $\sim 0.3-0.5 \mathrm{~K}$, not shown). This may be caused by small differences in the implementation of the R98 gas absorption and/or the radiative transfer code. This issue is currently under investigation, though its understanding goes beyond the scope of this paper. Comparing Figs. 4 and 5, we notice slightly larger differences (by $0.1-0.2 \mathrm{~K}$ ) in the RTTOVgb vs. ARTS than in the RTTOV-gb vs. R98 tests. We attribute this to the fact that RTTOV-gb is totally independent of ARTS and moreover to the specific profile dataset, which likely introduces biases with respect to the RTTOV-gb training climatology. Note that TB differences for all the channels are of the same order of magnitude as those found between ARTS and the original nadir-looking RTTOV (Buehler et al., 2006). This demonstrates comparable capabilities between RTTOV-gb and the original version of RTTOV. The rms TB differences between RTTOV-gb and ARTS at $90^{\circ}$ elevation are within $0.5 \mathrm{~K}$, thus below the uncertainty associated with
TB observations. From the three tests above, we can conclude that in the elevation angle range from 90 to $10^{\circ}$, the forward model error due to the use of the fast RT with respect to the reference LBL model is within the instrument uncertainty. This confirms that RTTOV-gb can be safely deployed in place of an LBL model into variational assimilation schemes.

\subsection{Comparison with real observations}

Another way to evaluate RTTOV-gb is to compare TB simulated from radiosonde profiles with TB measured by a nearly co-located microwave radiometer. This comparison provides an end-to-end evaluation of the model, though radiosonde drift, MWR calibration, finite beamwidth, discretization, and instrumental noise all contribute to the total uncertainty. Nevertheless, observations minus background model $(\mathrm{O}-\mathrm{B})$ differences are the primary input for direct radiance assimilation into an NWP model, and thus need to be investigated and understood. For this analysis, we exploit a dataset of 365 radiosonde profiles collected over Bordeaux from April to October 2014, together with the nearly simultaneous TB observed by a ground-based microwave radiometer (HAT- 
Table 3. Statistics for the comparison between RTTOV-gb and the line-by-line model R98 at MP-3000A channels with the best RTTOV training configuration, for both the dependent (top) and independent (bottom) profile set (R98 minus RTTOV-gb). MP3000A channel number (Chan no.), the channel central frequency, bias, and rms at elevation angles $90,30,19$, and $10^{\circ}$ are reported.

\begin{tabular}{|c|c|c|c|c|c|c|c|c|c|}
\hline \multirow[b]{3}{*}{ Chan no. } & \multirow[b]{3}{*}{ Frequency $(\mathrm{GHz})$} & \multicolumn{8}{|c|}{ Dependent profile set } \\
\hline & & \multicolumn{4}{|c|}{ Bias $(\mathrm{K})$} & \multicolumn{4}{|c|}{$\operatorname{rms}(\mathrm{K})$} \\
\hline & & $90^{\circ}$ & $30^{\circ}$ & $19^{\circ}$ & $10^{\circ}$ & $90^{\circ}$ & $30^{\circ}$ & $19^{\circ}$ & $10^{\circ}$ \\
\hline 1 & 22.23 & -0.016 & 0.027 & -0.004 & -0.319 & 0.059 & 0.047 & 0.044 & 0.376 \\
\hline 2 & 22.50 & -0.015 & 0.026 & -0.004 & -0.321 & 0.058 & 0.047 & 0.044 & 0.378 \\
\hline 3 & 23.03 & -0.009 & 0.025 & -0.005 & -0.318 & 0.053 & 0.045 & 0.042 & 0.370 \\
\hline 4 & 23.83 & 0.002 & 0.020 & -0.007 & -0.313 & 0.043 & 0.040 & 0.038 & 0.357 \\
\hline 5 & 25.00 & 0.010 & 0.014 & -0.007 & -0.300 & 0.039 & 0.037 & 0.037 & 0.346 \\
\hline 6 & 26.23 & 0.011 & 0.010 & -0.006 & -0.284 & 0.037 & 0.036 & 0.036 & 0.343 \\
\hline 7 & 28.00 & 0.011 & 0.008 & -0.005 & -0.270 & 0.036 & 0.037 & 0.037 & 0.3500 \\
\hline 8 & 30.00 & 0.011 & 0.006 & -0.004 & -0.266 & 0.038 & 0.040 & 0.040 & 0.366 \\
\hline 9 & 51.25 & 0.024 & 0.018 & 0.013 & -0.088 & 0.177 & 0.171 & 0.128 & 0.104 \\
\hline 10 & 51.76 & 0.024 & 0.019 & 0.013 & -0.056 & 0.189 & 0.164 & 0.111 & 0.066 \\
\hline 11 & 52.28 & 0.025 & 0.019 & 0.012 & -0.029 & 0.203 & 0.149 & 0.085 & 0.034 \\
\hline 12 & 52.80 & 0.029 & 0.020 & 0.008 & -0.011 & 0.207 & 0.116 & 0.052 & 0.014 \\
\hline 13 & 53.37 & 0.019 & 0.017 & 0.002 & -0.002 & 0.181 & 0.068 & 0.022 & 0.005 \\
\hline 14 & 53.85 & 0.012 & 0.007 & 0.001 & 0.000 & 0.120 & 0.029 & 0.010 & 0.003 \\
\hline 15 & 54.40 & 0.006 & -0.000 & 0.001 & 0.000 & 0.055 & 0.012 & 0.006 & 0.002 \\
\hline 16 & 54.94 & 0.004 & 0.001 & 0.000 & 0.000 & 0.023 & 0.007 & 0.003 & 0.001 \\
\hline 17 & 55.50 & 0.002 & 0.001 & 0.000 & -0.000 & 0.013 & 0.004 & 0.002 & 0.000 \\
\hline 18 & 56.02 & 0.001 & 0.000 & 0.000 & -0.000 & 0.009 & 0.003 & 0.001 & 0.000 \\
\hline 19 & 56.66 & 0.001 & 0.000 & 0.000 & -0.000 & 0.007 & 0.002 & 0.000 & 0.000 \\
\hline 20 & 57.29 & 0.001 & 0.000 & -0.000 & -0.000 & 0.005 & 0.001 & 0.000 & 0.000 \\
\hline 21 & 57.96 & 0.001 & 0.000 & -0.000 & -0.000 & 0.004 & 0.001 & 0.000 & 0.000 \\
\hline 22 & 58.80 & 0.000 & 0.000 & -0.000 & -0.000 & 0.004 & 0.001 & 0.000 & 0.000 \\
\hline
\end{tabular}

Independent profile set

\begin{tabular}{lrrrrr|rrrr}
\hline & & & \multicolumn{2}{c}{ Bias $(\mathrm{K})$} & & \multicolumn{2}{c}{ rms $(\mathrm{K})$} \\
\hline Chan no. & Frequency $(\mathrm{GHz})$ & $90^{\circ}$ & $30^{\circ}$ & $19^{\circ}$ & $10^{\circ}$ & $90^{\circ}$ & $30^{\circ}$ & $19^{\circ}$ & $10^{\circ}$ \\
\hline 1 & 22.23 & -0.008 & 0.022 & -0.003 & -0.284 & 0.049 & 0.046 & 0.042 & 0.157 \\
2 & 22.50 & -0.008 & 0.021 & -0.005 & -0.279 & 0.048 & 0.046 & 0.043 & 0.158 \\
3 & 23.03 & -0.002 & 0.020 & -0.006 & -0.277 & 0.042 & 0.045 & 0.042 & 0.154 \\
4 & 23.83 & 0.007 & 0.017 & -0.008 & -0.274 & 0.035 & 0.044 & 0.045 & 0.164 \\
5 & 25.00 & 0.012 & 0.011 & -0.009 & -0.263 & 0.033 & 0.043 & 0.051 & 0.206 \\
6 & 26.23 & 0.011 & 0.007 & -0.009 & -0.247 & 0.031 & 0.041 & 0.052 & 0.236 \\
7 & 28.00 & 0.010 & 0.004 & -0.008 & -0.232 & 0.031 & 0.040 & 0.053 & 0.257 \\
8 & 30.00 & 0.008 & 0.002 & -0.009 & -0.228 & 0.033 & 0.043 & 0.057 & 0.273 \\
9 & 51.25 & -0.005 & -0.018 & -0.016 & -0.094 & 0.156 & 0.160 & 0.128 & 0.067 \\
10 & 51.76 & -0.005 & -0.014 & -0.010 & -0.061 & 0.162 & 0.149 & 0.105 & 0.039 \\
11 & 52.28 & -0.005 & -0.009 & -0.004 & -0.037 & 0.170 & 0.131 & 0.077 & 0.020 \\
12 & 52.80 & -0.004 & 0.000 & -0.001 & -0.015 & 0.169 & 0.098 & 0.044 & 0.015 \\
13 & 53.37 & -0.003 & 0.007 & -0.003 & -0.005 & 0.145 & 0.056 & 0.021 & 0.015 \\
14 & 53.85 & -0.002 & 0.002 & -0.002 & -0.002 & 0.097 & 0.026 & 0.015 & 0.012 \\
15 & 54.40 & 0.000 & -0.002 & -0.001 & -0.001 & 0.047 & 0.015 & 0.011 & 0.007 \\
16 & 54.94 & 0.002 & 0.000 & -0.000 & -0.000 & 0.023 & 0.011 & 0.008 & 0.003 \\
17 & 55.50 & 0.002 & 0.000 & 0.000 & -0.000 & 0.016 & 0.007 & 0.005 & 0.001 \\
18 & 56.02 & 0.002 & 0.000 & 0.000 & -0.000 & 0.013 & 0.005 & 0.003 & 0.001 \\
19 & 56.66 & 0.001 & 0.000 & 0.000 & 0.000 & 0.010 & 0.004 & 0.002 & 0.000 \\
20 & 57.29 & 0.000 & 0.000 & 0.000 & 0.000 & 0.009 & 0.003 & 0.001 & 0.000 \\
21 & 57.96 & 0.000 & 0.000 & 0.000 & 0.000 & 0.008 & 0.002 & 0.000 & 0.000 \\
22 & 58.80 & 0.000 & 0.000 & 0.000 & 0.000 & 0.007 & 0.002 & 0.000 & 0.000 \\
\hline & & & & & & & & &
\end{tabular}



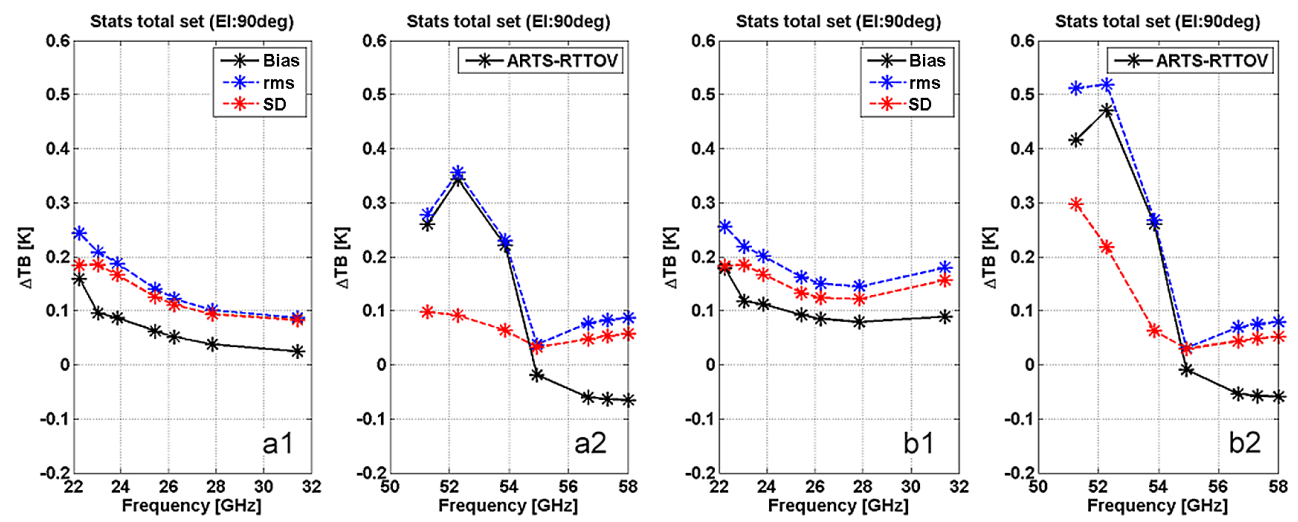

Figure 5. Bias (black solid line), standard deviation (red dashed line), and rms (blue dashed line) of TB differences between RTTOV-gb and the reference radiative transfer model ARTS (Eriksson and Buehler, 2015), for both clear (a1-2) and cloudy (b1-2) sky conditions (ARTS minus RTTOV-gb). Panels (a1, b1-a2, b2) are for K- and V-band channels, respectively. All panels report results at $90^{\circ}$ elevation angle.

PRO) operated at the radiosonde launching site. The dataset was first reduced to clear-sky conditions. To be conservative, clear-sky conditions have been selected using three-fold screening, based on (i) ceilomenter cloud base height (CBH), (ii) sky infrared temperature $\left(T_{\mathrm{IR}}\right)$, and (iii) 20 min standard deviation of liquid water path $\left(\sigma_{\mathrm{LWP}}\right)$ from HATPRO. Thus, periods with $\mathrm{CBH}$ below maximum range $(8000 \mathrm{~m})$, $T_{\mathrm{IR}}>-30^{\circ} \mathrm{C}$, or $\sigma_{\mathrm{LWP}}>10^{-2} \mathrm{~kg} \mathrm{~m}^{-3}$ were rejected. Moreover, cases with integrated water vapor differences between microwave radiometer and radiosonde profiles larger than $1 \mathrm{~mm}$ have been discarded in order to reduce instrumental uncertainties involved in the comparison. After this screening, only 23 profiles remained for the analysis. Bias, standard deviation, and rms differences between TB observed by the microwave radiometer and simulated with both RTTOV-gb and ARTS are shown in Fig. 6. With respect to the MWR observations, RTTOV-gb shows bias from $0.02 \mathrm{~K}$ at $22.24 \mathrm{GHz}$ to $0.5 \mathrm{~K}$ at $23.84 \mathrm{GHz}$ in the $\mathrm{K}$-band and from 0.16 to $0.31 \mathrm{~K}$ in the V-band opaque channels. The rms errors range from 0.90 to $0.47 \mathrm{~K}$ in the K-band and from 0.41 to $0.64 \mathrm{~K}$ in the V-band opaque channels. Larger bias is found at V-band transparent channels: $1-2 \mathrm{~K}$ at $51.26 \mathrm{GHz}$ and $4-5 \mathrm{~K}$ at $52.28 \mathrm{GHz}$ with either RTTOV-gb or ARTS simulations. Note that RTTOV$\mathrm{gb}$ and ARTS show similar statistics with respect to MWR observations. This result is very important as it suggests that forward model errors due to the fast model approximation are not dominant. Note that bias values of the same order of magnitude for the $51-54 \mathrm{GHz}$ range were previously reported (Hewison et al., 2006; Löhnert and Maier, 2012; Martinet et al., 2015; Blumberg et al., 2015), employing MWRs of different types and manufacturers. This may be attributed to a combination of uncertainties from instrument calibration and gas absorption models. In fact, semi-transparent channels (as in the $51-54 \mathrm{GHz}$ range) suffer from larger calibration uncertainties due to the lack of a close referencetemperature calibration point. In addition, their response is influenced by the water vapor continuum and oxygen line
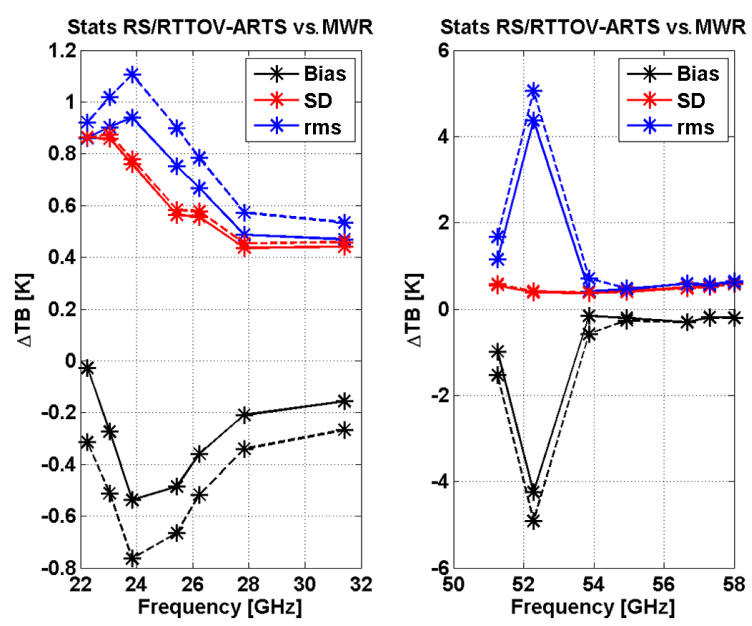

Figure 6. Bias (black line), standard deviation (red line), and rms (blue line) of differences between TB measured with the microwave radiometer and TB simulated from radiosonde profiles respectively with RTTOV-gb (solid lines) and the reference radiative transfer model ARTS (dashed lines), both for clear-sky conditions at $90^{\circ}$ elevation angle (measurements minus simulations).

coupling, which contribute significantly to the uncertainties because their parametrization is extrapolated from laboratory measurements to typical atmospheric conditions. It is beyond the scope of this paper to investigate spectroscopy issues, but our results support previous evidence and point to the need for further lab measurements (Boukabara et al., 2005; Cadeddu et al., 2007). Considering that $\mathrm{O}-\mathrm{B}$ systematic differences are usually evaluated and removed before assimilating data into NWP, we believe that statistics in Fig. 6 support the safe use of RTTOV-gb for direct radiance assimilation of MWR TB into NWP models. 

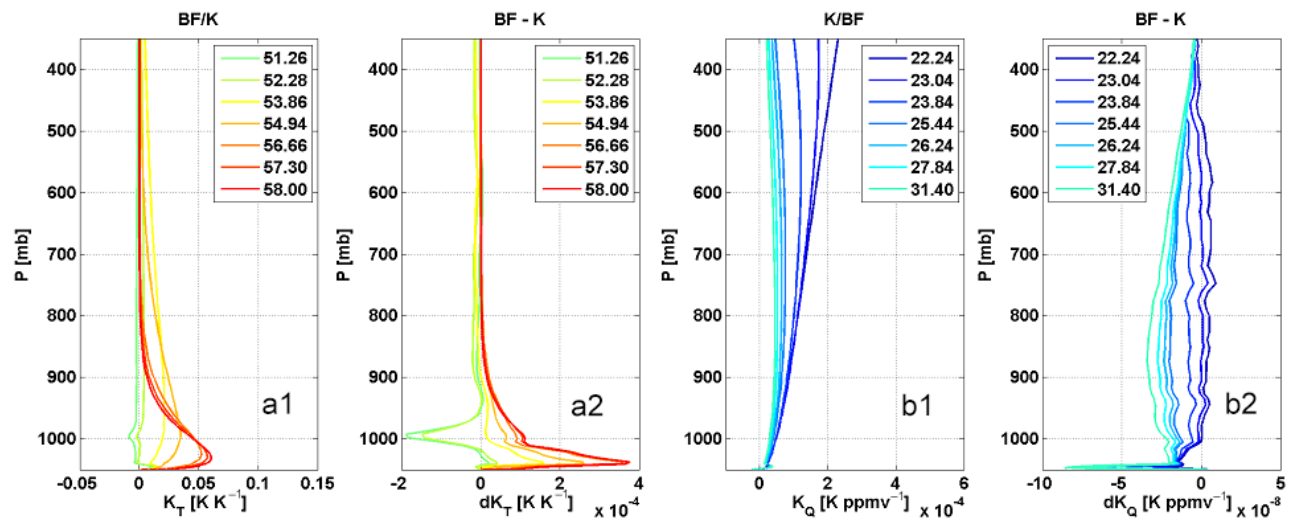

Figure 7. Jacobians calculated with the RTTOV-gb BF method and K-module. (a1) Temperature Jacobians for V-band channels; (b1) absolute humidity for K-band channels. Note that the BF method (solid line) and K-module (dashed line) are not distinguishable as they nearly completely overlap. Panels (a2, b2) show Jacobian differences between BF and K, for temperature and absolute humidity respectively.

\subsection{Comparison of Jacobians}

After testing the RTTOV-gb direct module, the RTTOV-gb Jacobians calculation needs to be tested in order to provide a complete tool for a fast and safe MWR data assimilation. First, a consistency test of the Jacobians calculated with TL-, AD-, and K-modules of RTTOV-gb has been performed to ensure the correctness of the TL/AD/K coding modified for a ground-based perspective. The test resulted in nearly the same Jacobians for TL, AD-, and K-modules. Subsequently, the temperature and humidity Jacobians calculated with the RTTOV-gb K-module have been compared with those computed with the brute force (BF) method for a specific cloudysky profile. The BF method calculates the Jacobian by finite differences by calling the direct module multiple times after perturbing each individual input profile variable. The consistency of K-module with BF was confirmed using the RTTOV test suite (Brunel and Hocking, 2014), bearing in mind that some small differences between the Jacobians are expected. Figure 7 shows the temperature and absolute humidity Jacobians for the V- and K-bands channels. The Jacobians computed with RTTOV-gb BF and K-module are almost identical with differences smaller than $1 \%$. As expected, the TB sensitivity to atmospheric temperature is higher in the low troposphere, especially in the PBL, and it increases with frequency in the spectral range between 51 and $58 \mathrm{GHz}$. Between 22 and $31 \mathrm{GHz}$, the sensitivity of the TBs to water vapor is almost independent of altitude and decreases with increasing frequency.

The Jacobians for cloud liquid water (CLW) are needed when cloudy-sky conditions are considered. Figure 8 shows a comparison of CLW Jacobians calculated with the RTTOV$\mathrm{gb}$ K-module and the BF method. Similar to temperature and humidity, they are found to be almost identical (differences smaller than $0.1 \%$, likely due to truncation errors). As expected, the TB sensitivity to CLW increases with frequency in the K-band, while it decreases with frequency in the V-
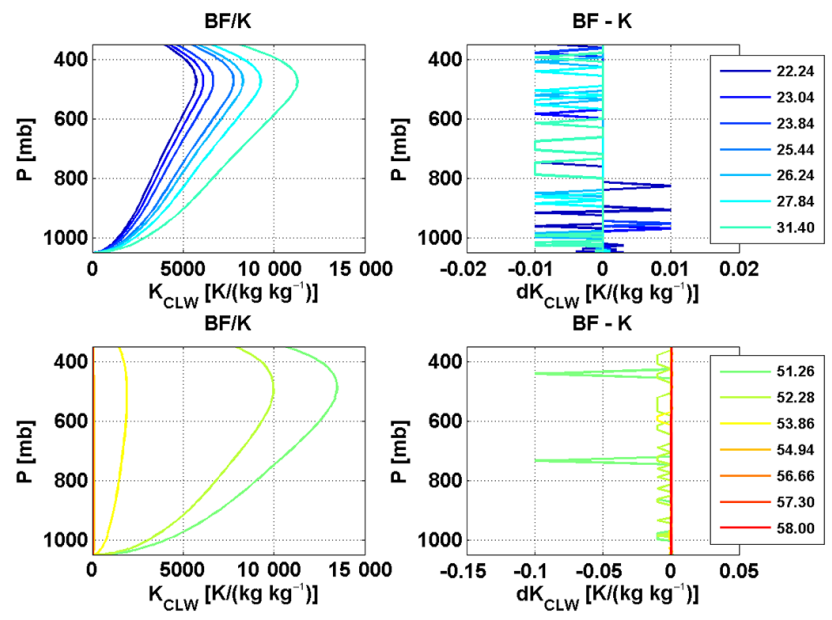

Figure 8. Cloud liquid water Jacobians calculated with the RTTOVgb BF method and K-module (left) and Jacobian differences between BF and K (right), for K-band (top) and V-band (bottom) channels, respectively. Note that the BF method (solid) and K-module (dashed) are not distinguishable as they nearly completely overlap.

band due to the increasingly dominant oxygen absorption. TBs are sensitive to CLW at all levels up to $322 \mathrm{hPa}$ (about $10 \mathrm{~km}$ ), where RTTOV, and thus also RTTOV-gb, have set their upper limit for non-zero CLW.

For a completely independent test, Jacobians calculated with the RTTOV K-module have been compared with those computed with the reference radiative transfer model ARTS. ARTS Jacobians are derived from a semi-analytical expression described in Eriksson and Buehler (2015). As shown in Fig. 9, temperature and humidity Jacobians from ARTS and RTTOV-gb are found to be almost identical, either for $\mathrm{K}$-band and V-band channels, with differences smaller than $3 \%$ for temperature and $5 \%$ for humidity. Figure 10 shows the comparison of CLW Jacobians from ARTS and RTTOV- 

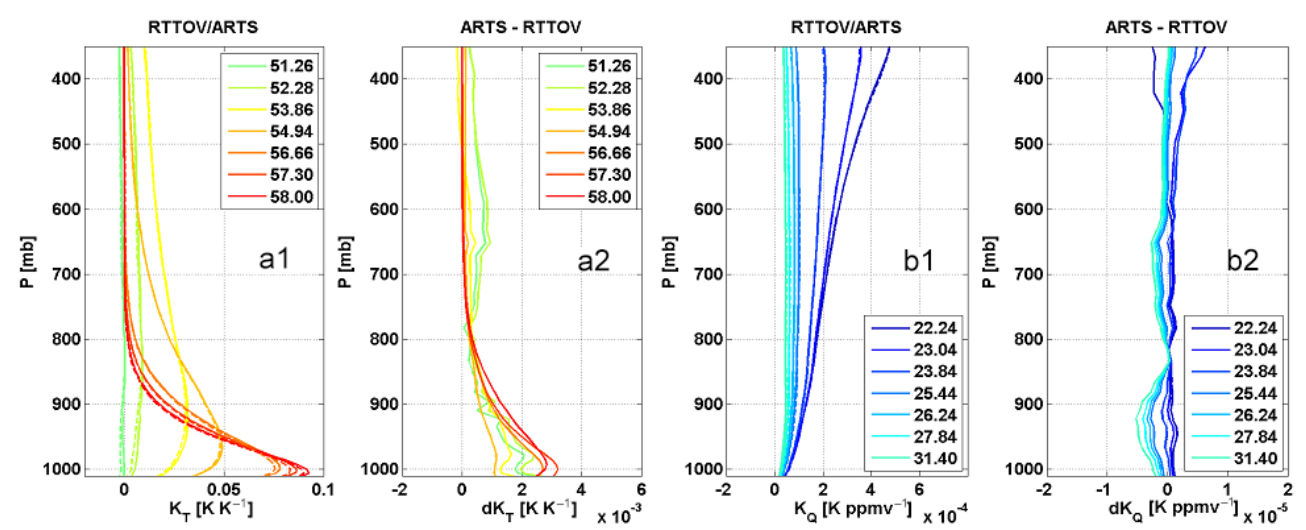

Figure 9. As in Fig. 7, but for Jacobians calculated with ARTS (solid line) and the RTTOV-gb K-module (dashed line). Panels (a2, b2) show Jacobian differences between ARTS and RTTOV-gb K-module, for temperature and absolute humidity respectively.
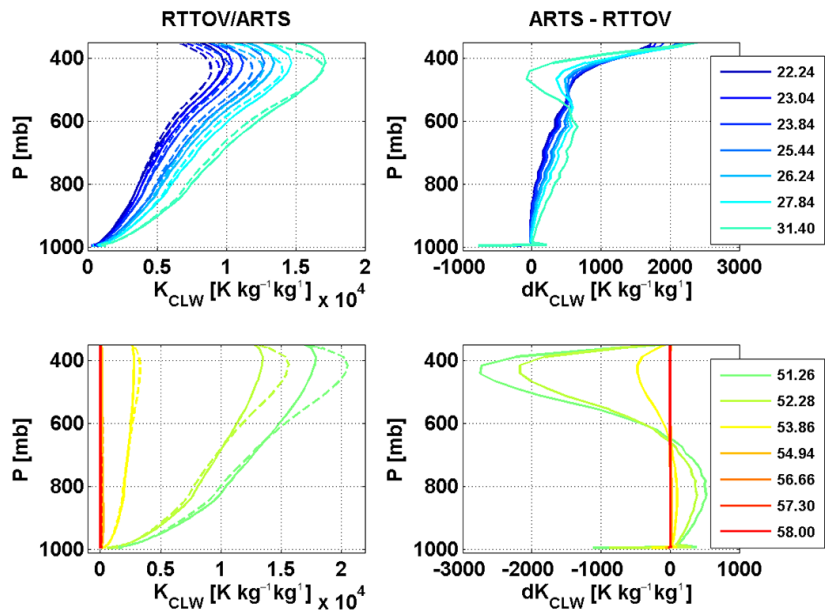

Figure 10. As in Fig. 8, but for Jacobians calculated with ARTS (solid line) and the RTTOV-gb K-module (dashed line).

$\mathrm{gb}$. These are similar to each other, both in shape and order of magnitude, from the surface up to $322 \mathrm{hPa}$ (RTTOV cloud limit). However, differences of about $10 \%$ occur around $450 \mathrm{hPa}$, particularly at transparent channels $(31,51$, and $52 \mathrm{GHz}$ ). These are likely due to small differences in the liquid water absorption models in ARTS and in RTTOV-gb, as mentioned above in Sect. 3.1. However, for a typical CLW profile, these model differences lead to small TB differences (order of $0.1 \mathrm{~K}$ ) and are thus deemed as negligible.

\subsection{D-Var application}

Finally, RTTOV-gb has been tested as a forward model within a one-dimensional variational (1D-Var) scheme. For this purpose, the 1D-Var software package provided by the NWPSAF (Weston, 2014) has been adapted in the framework of the COST Action TOPROF to exploit RTTOV-gb. Among other modifications, the 1D-Var tool has been modified to al- low the assimilation of observations at different elevation angles for the same instrument. The 1D-Var approach searches the atmospheric state $\boldsymbol{x}$ that minimizes both the distance to the background $\boldsymbol{x}_{\mathrm{b}}$ and the observation $\boldsymbol{y}$. The cost function $J$ needs to be minimized, modifying the different variables defined in the control vector $\boldsymbol{x}$ (Cimini et al., 2010):

$$
\begin{aligned}
J & =\frac{1}{2}[\boldsymbol{y}-H(\boldsymbol{x})]^{\mathrm{T}} R^{-1}[\boldsymbol{y}-H(\boldsymbol{x})] \\
& +\frac{1}{2}\left[\boldsymbol{x}-\boldsymbol{x}_{\mathrm{b}}\right]^{\mathrm{T}} \mathbf{B}^{-1}\left[\boldsymbol{x}-\boldsymbol{x}_{\mathrm{b}}\right] .
\end{aligned}
$$

Here $\mathbf{B}$ represents the background-error covariance matrix and $\mathbf{R}$ the observation error covariance matrix. $H$ represents the observation operator, in our case RTTOV-gb. The background profile comes from a short-range forecast of an NWP model or from a co-located radiosonde. Here, $\boldsymbol{x}_{\mathrm{b}}$ is a $3 \mathrm{~h}$ forecast from the French convective-scale model AROME. The Jacobians needed to minimize the cost function $J$ are calculated with the RTTOV-gb K-module.

The aim is to retrieve temperature and humidity profiles and column-integrated liquid water path from MWR observations through a 1D-Var retrieval approach exploiting RTTOV-gb. According to this aim, an Observing System Simulation Experiment (OSSE) was set up with 224 AROME analyses profiles in February 2015 over the Alps with the new horizontal grid mesh of $1.3 \mathrm{~km}$. These analyses are made of 90-level pressure, temperature, specific humidity, and liquid water content profiles, typical of an alpine valley and mountainous region in winter. Both clearand cloudy-sky conditions are considered. Starting from the AROME unperturbed profiles (the "truth"), background profiles are created by perturbing the initial AROME profiles according to the background error covariance matrix $\mathbf{B}$. In this study, the $\mathbf{B}$ matrix was computed from an AROME ensemble assimilation system, following the approach used to derive this matrix operationally at Météo France (Brousseau et al., 2011). By applying RTTOV-gb to the unperturbed AROME, observations are created by adding synthetic ob- 
servation errors to the RTTOV-gb simulations. The synthetic random errors are assumed to follow a diagonal $\mathbf{R}$ matrix with reasonable standard deviations, i.e., $\sim 0.2-1.0 \mathrm{~K}$ depending on channels (Hewison, 2007).

In clear-sky conditions, temperature and specific humidity are used as control variables in the 1D-Var. A comparison between temperature and humidity retrievals obtained with 1D-Var and the corresponding unperturbed and background profiles for two retrieval examples are shown in Fig. 11. As expected, the 1D-Var retrievals are closer to the truth than the background profiles. In this case 1-D-Var provides an improvement with respect to the background in the first $2 \mathrm{~km}$ for temperature and in the first $4 \mathrm{~km}$ for humidity, which is encouraging for future data assimilation experiments. A comprehensive evaluation of RTTOV-gb plus 1D-Var for data assimilation using real MWR observations will be the subject of future work.

Here, we just underline that the main advantage of RTTOV-gb with respect to LBL models is the considerably lower computation time. Of course the priority of LBL models is more accuracy than speed, though settings may be tuned to improve the computation performances. Although a detailed analysis on computation speed goes beyond the scope of this paper, we found that RTTOV-gb is faster than our implementation of ARTS (Martinet et al., 2015) for both the direct and Jacobian calculations. Moreover, our tests demonstrate that the computation time for Jacobians is shorter by a factor of 8 for the RTTOV-gb K-module than for the direct module with the brute force method.

\section{Summary}

Version 11.2 of the fast radiative transfer model RTTOV, developed for space-borne sensors, has been successfully modified to simulate ground-based microwave radiometer observations. In addition to the direct module, which allows ground-based MWR observations to be simulated, the TL-, AD-, and K-modules of RTTOV have been modified in order to provide temperature, humidity, and cloud liquid water Jacobians for the ground-based perspective. We introduced the ground-based version of RTTOV, called RTTOV$\mathrm{gb}$, and demonstrated its potential for fast MWR TB simulations from thermodynamic profiles. RTTOV-gb has been validated against accurate, but less time-efficient, reference lineby-line models and real MWR observations. Results demonstrate its applicability as a forward model within a variational scheme for fast and safe MWR data assimilation into NWP models. It is believed that the direct assimilation of TB, instead of retrieved profiles, may improve the impact of MWR observations for temperature and humidity profiles analysis in the first few kilometers from the ground, where MWRs provide the maximum information content.

The performance of RTTOV-gb has been validated by comparison with TB simulated with the line-by-line model
R98 (Rosenkranz, 1998), the same model as used for the RTTOV training phase. For both dependent and independent profile sets, rms errors are below the typical TB uncertainty of ground-based MWRs $(\sim 0.5 \mathrm{~K})$, ranging from a maximum of $0.06 \mathrm{~K}$ for the water vapor band to $0.025 \mathrm{~K}$ for the V-band opaque channels. Larger discrepancies are observed at the transparent V-band channels (51 and $52 \mathrm{GHz}$ ), with an rms within $0.20 \mathrm{~K}$, and at elevation angle $10^{\circ}$. TBs simulated with RTTOV-gb from AROME analyses have also been compared with those simulated with the reference line-by-line model ARTS. At $90^{\circ}$ elevation, for both clear- and cloudy-sky conditions TB differences do not exceed $0.25 \mathrm{~K}$ in terms of biases and rms at all HATPRO channels except for the transparent V-band channels $51-52 \mathrm{GHz}$ (up to $0.5 \mathrm{~K}$ in cloudysky conditions). Finally, RTTOV-gb has been validated by radiosonde-derived TB with real nearly collocated MWR observations. In this case, the rms error increases with respect to the RTTOV-gb/LBL comparisons, ranging from 0.90 to $0.47 \mathrm{~K}$ in the K-band and from 0.41 to $0.64 \mathrm{~K}$ in the V-band opaque channels. Larger discrepancies were found at V-band transparent channels, which may be explained by calibration and gas absorption uncertainties. However, the statistics of RTTOV-gb and ARTS simulations with respect to MWR observations are similar for each channel, suggesting that forward model errors due to the fast model approximation are not dominant. Temperature, humidity, and cloud liquid water Jacobians computed with RTTOV-gb K-modules were found to be similar in shape and magnitude with those calculated with the brute force method or with the ARTS model.

Finally, RTTOV-gb has been tested as a forward model within a 1D-Var software package in an OSSE to improve AROME thermodynamic profiles estimated by directly assimilating synthetic MWR TB. For both temperature and humidity profiles, the $1 \mathrm{D}$-Var considerably improves the retrievals with respect to the background, in the first few kilometers from the ground. Concerning the computation speed, RTTOV-gb with K-module is found to be 8 times faster in computing Jacobians than the brute force method. As expected, RTTOV-gb is demonstrated to be faster than the lineby-line models such as ARTS for both the direct and the Jacobians calculation.

Ultimately, this analysis confirms that RTTOV-gb is able to correctly simulate ground-based MWR radiance and to reproduce reasonable temperature, humidity, and cloud liquid water Jacobians. In conclusion, RTTOV-gb is well suited for serving as a forward model in a variational data assimilation scheme for a direct, safe, and fast NWP data assimilation of real MWR radiance observations. As from the user perspective, RTTOV-gb works exactly the same as RTTOV; its implementation and maintenance shall require minimal technical overheads at those NWP centers already using RTTOV. This shall facilitate the road towards the data assimilation of ground-based MWRs worldwide. 

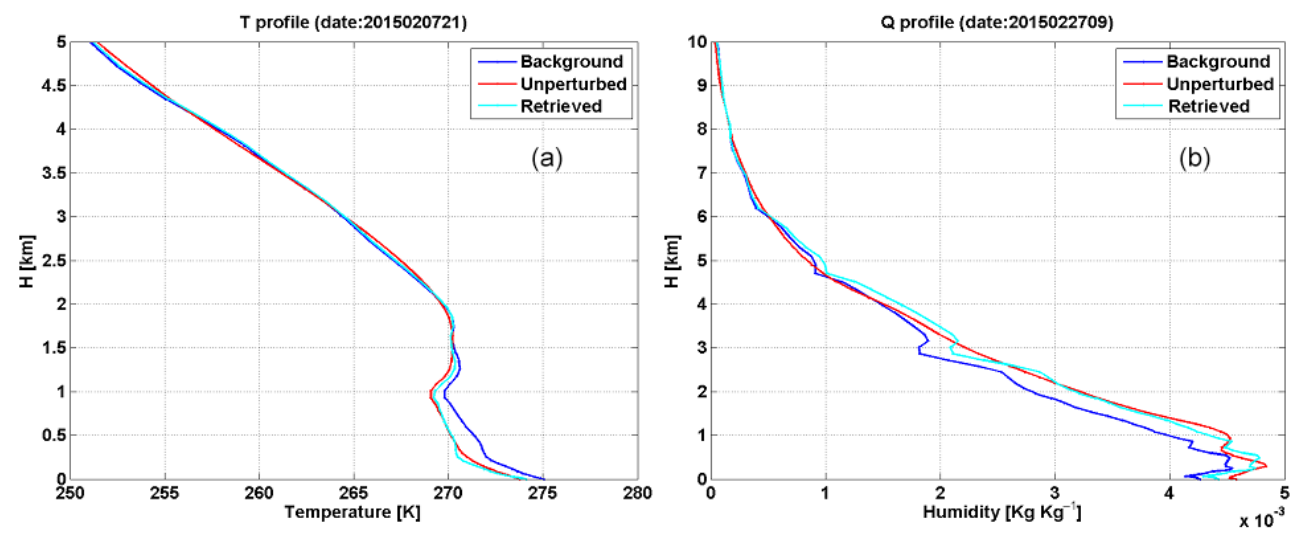

Figure 11. Temperature (a) and humidity (b) profiles of background (blue line), truth (red line), and 1D-Var retrievals (cyan line) for two clear-sky profiles.

\section{Code and data availability}

The original RTTOV v11.2 can be obtained via the request form on the NWPSAF website (NWPSAF, 2013; http: //nwpsaf.eu/site/software/rttov/rttov-v11/).

The efforts for adapting RTTOV to ground-based observations started within the COST (http://www.cost.eu/) action ES1202 (EG-CLIMET) and have been continued within the COST action ES1303 (TOPROF, http://www.toprof.eu/). The modifications needed to adapt the radiative transfer equation from the satelliteto the ground-based perspective have been made in the subroutine src/main/rttov_integrate.F90. The RTTOV subroutines that have been modified in RTTOV-gb to reverse the way that transmittances and optical depths are initialized and accumulated are src/main/rttov_transmit.F90 and src/main/rttov_opdep.F90 respectively. The calculation of the predictors 7 for the ground-based perspective has been adapted in the subroutine src/main/rttov_profaux.F90. Modifications made in the direct module of RTTOV v11.2 code have been imported in the corresponding TL-, AD-, and K-modules' subroutines (i.e., rttov_integrate_tl.F90, rttov_integrate_ad.F90, rttov_integrate_k.F90; rttov_transmit_tl.F90, rttov_transmit_ad.F90, rttov_transmit_k.F90; rttov_opdep_1.F90, rttov_opdep_ad.F90, rttov_opdep_k.F90). The conditions of release of RTTOV-gb are currently under discussion among NWPSAF and COST action TOPROF. This may happen through an integration of RTTOV-gb into future RTTOV releases or as a stand-alone package disseminated through the TOPROF website.

All the information needed to download the ARTS code can be found on the website: http://www.radiativetransfer. org/.

The NWPSAF profiles, from which we interpolated the profile sets used for the RTTOV-gb training and independent test, are available at https://nwpsaf.eu/deliverables/rtm/ profile_datasets.html.

The AROME analyses used for ARTS/RTTOV$\mathrm{gb}$ comparison and 1D-Var application, and the MWR/radiosondes dataset used for the validation against real MWR measurement, can be obtained by emailing pauline.martinet@meteo.fr. 


\section{Appendix A}

The predictors $X_{k j}$ introduced in Sect. 2 are functions of the absorbing gas, the zenith angle $\theta$, the pressure, temperature, and water vapor mixing ratio profiles, and finally the reference temperature and water vapor mixing ratio profiles (i.e., the average of the training profile set). These are defined in Matricardi et al. (2001) and briefly summarized below. Introducing at each fixed level $j$ the pressure $P^{\text {prof }}(j)$, the temperature, and the water vapor mixing ratio $T^{\text {prof }}(j)$ and $W^{\text {prof }}(j)$, and the corresponding reference $T^{\text {ref }}(j)$ and $W^{\text {ref }}(j)$, the following variables are defined:

$$
\begin{aligned}
& T(j)=\left[T^{\text {prof }}(j)+T^{\text {prof }}(j+1)\right] / 2 \\
& T^{*}(j)=\left[T^{\text {ref }}(j)+T^{\text {ref }}(j+1)\right] / 2 \\
& W(j)=\left[W^{\text {prof }}(j)+W^{\text {prof }}(j+1)\right] / 2 \\
& W^{*}(j)=\left[W^{\text {ref }}(j)+W^{\text {ref }}(j+1)\right] / 2 \\
& P(j)=\left[P^{\text {prof }}(j)+P^{\text {prof }}(j+1)\right] / 2 \\
& T_{r}(j)=T(j) / T^{*}(j) \\
& \delta T(j)=T(j)-T^{*}(j) \\
& W_{r}(j)=W(j) / W^{*}(j) \\
& T_{w}(j)=\sum_{l=N-1}^{j} P(l+1)[P(l+1)-P(l)] T_{r}(l+1),
\end{aligned}
$$

with $T_{w}(j=N)=0$ at the surface.

$$
\begin{gathered}
W_{w}(j)=\left\{\sum_{l=N-1}^{j} P(l+1)[P(l+1)-P(l)] W(l)\right\} / \\
\left\{\sum_{l=N-1}^{j} P(l+1)[P(l+1)-P(l)] W^{*}(l)\right\}
\end{gathered}
$$

The RTTOV predictors 7 are derived from the variables above as listed in Table A1.
Table A1. Predictors 7 used for mixed gases and water vapor (after Matricardi et al., 2001).

\begin{tabular}{lll}
\hline Predictor 7 & Mixed gases & Water vapor \\
\hline$X_{1, j}$ & $\sin (\theta)$ & $\sin ^{2}(\theta) W_{r}^{2}(j)$ \\
$X_{2, j}$ & $\sin ^{2}(\theta)$ & $\left(\sin (\theta) W_{w}(j)\right)^{2}$ \\
$X_{3, j}$ & $\sin (\theta) T_{r}(j)$ & $\left(\sin (\theta) W_{w}(j)\right)^{4}$ \\
$X_{4, j}$ & $\sin (\theta) T_{r}^{2}(j)$ & $\sin (\theta) W_{r}(j) \delta T(j)$ \\
$X_{5, j}$ & $T_{r}(j)$ & $\sqrt{\sin (\theta) W_{r}(j)}$ \\
$X_{6, j}$ & $T_{r}^{2}(j)$ & $\sqrt[4]{\sin (\theta) W_{r}(j)}$ \\
$X_{7, j}$ & $\sin (\theta) T_{w}(j)$ & $\sin (\theta) W_{r}(j)$ \\
$X_{8, j}$ & $\sin (\theta) \frac{T_{w}(j)}{T_{r}(j)}$ & $\left(\sin (\theta) W_{r}(j)\right)^{3}$ \\
$X_{9, j}$ & $\sqrt{\sin (\theta)}$ & $\left(\sin (\theta) W_{r}(j)\right)^{4}$ \\
$X_{10, j}$ & $\sqrt{\sin (\theta)} \sqrt[4]{T_{w}(j)}$ & $\sin (\theta) W_{r}(j) \delta T(j)|\delta T(j)|$ \\
$X_{11, j}$ & 0 & $\left(\sqrt{\left.\sin (\theta) W_{r}(j)\right) \delta T(j)}\right.$ \\
$X_{12, j}$ & 0 & $\frac{\left(\sin (\theta) W_{r}(j)\right)^{2}}{W_{w}}$ \\
$X_{13, j}$ & 0 & $\frac{\sqrt{\sin (\theta) W_{r}(j)} W_{r}(j)}{W_{w}(j)}$ \\
$X_{14, j}$ & 0 & $\sin (\theta) \frac{W_{r}^{2}(j)}{T_{r}(j)}$ \\
$X_{15, j}$ & 0 & $\sin (\theta) \frac{W_{r}^{2}(j)}{T_{r}^{4}(j)}$ \\
\hline
\end{tabular}


Acknowledgements. This work has been stimulated through the COST Action ES1303 (TOPROF), supported by COST (European Cooperation in Science and Technology). Part of the work was supported by the EU H2020 project GAIA-CLIM (Ares(2014)3708963/Project 640276). The authors would like to acknowledge the NWPSAF and Met Office, in particular Peter Rayer, for providing support with RTTOV coding, and Météo France, for providing AROME analyses and measurements performed in the Bordeaux campaigns.

Edited by: K. Gierens

Reviewed by: two anonymous referees

\section{References}

Blumberg, W. G., Turner, D. D., Löhnert, U., and Castleberry, S.: Ground-Based Temperature and Humidity Profiling Using Spectral Infrared and Microwave Observations. Part II: Actual Retrieval Performance in Clear-Sky and Cloudy Conditions, J. Appl. Meteorol. Clim., 54, 2305-2319, doi:10.1175/JAMC-D15-0005.1, 2015.

Boukabara S. A., Clough, S. A., Moncet, J.-L., Krupnov, A. F., Tretyakov, M. Yu., and Parshin, V. V.: Uncertainties in the Temperature Dependence of the Line-Coupling Parameters of the Microwave Oxygen Band: Impact Study, IEEE T. Geosci. Remote Sens., 43, 1109-1114, doi:10.1109/TGRS.2004.839654, 2005.

Brousseau, P., Berre, L., Bouttier, F., and Desroziers, G.: Background error covariances for a convective scale data assimilation system: AROME 3D-Var, Q. J. Roy. Meteor. Soc., 137, 409-422, 2011.

Brunel, P. and Hocking, J.: RTTOV v11 Test Suite, Met Office Doc ID: NWPSAF-MO-TV-031, 2014.

Buehler, S. A., Eriksson, P., Kuhn, T., von Engeln, A., and Verdes, C.: ARTS, the Atmospheric Radiative Transfer Simulator, J. Quant. Spectrosc. Ra., 91, 65-93, doi:10.1016/j.jqsrt.2004.05.051, 2005.

Buehler, S. A., Courcoux, N., and John, V. O.: Radiative transfer calculations for a passive microwave satellite sensor: Comparing a fast model and a line-by-line model, J. Geophys. Res., 111, D20304, doi:10.1029/2005JD006552, 2006.

Cadeddu, M. P., Payne, V. H., Clough, S. A., Cady-Pereira, K., and Liljegren, J. C.: The effect of the oxygen line-parameter modeling on temperature and humidity retrievals from groundbased microwave radiometers, IEEE T. Geosci. Remote Sens., 45, 2216-2223, 2007.

Caumont, O., Cimini, D., Löhnert, U., Alados-Arboledas, L., Bleisch, R., Buffa, F., Ferrario, M. E., Haefele, A., Huet, T., Madonna, F., and Pace, G.: Assimilation of humidity and temperature observations retrieved from ground-based microwave radiometers into a convective-scale NWP model, Q. J. Roy. Meteor. Soc., doi:10.1002/qj.2860, 2016.

Cimini, D., Hewison, T. J., Martin, L., Güldner, J., Gaffard, C., and Marzano, F.: Temperature and humidity profile retrievals from groundbased microwave radiometers during TUC, Meteorol. Z., 15, 45-56, 2006.

Cimini, D., Westwater, E. R., and Gasiewski, A. J.: Temperature and Humidity Profiling in the Arctic Using Ground-Based
Millimeter-Wave Radiometry and 1DVAR, IEEE T. Geosci. Remote Sens., 48, 1381-1388, 2010.

Cimini, D., Campos, E., Ware, R., Albers, S., Graziano, G., Oreamuno, J., Joe, P., Koch, S., Cober, S., and Westwater, E.: Thermodynamic Atmospheric Profiling during the 2010 Winter Olympics Using Ground-based Microwave Radiometry, T. Geosci. Remote Sens., 49, 4959-4969, 2011.

Cimini D., Caumont, O., Löhnert, U., Alados-Arboledas, L., Bleisch, R., Fernández-Gálvez, J., Huet, T., Ferrario, M. E., Madonna, F., Maier, O., Nasir, F., Pace, G., and Posada, R.: An International Network of Ground-Based Microwave Radiometers for the Assimilation of Temperature and Humidity Profiles into NWP Models, Proceedings of 9th International Symposium on Tropospheric Profiling, ISBN 978-90-815839-4-7, L'Aquila, ITALY, 3-7 September 2012.

Cimini, D., Caumont, O., Löhnert, U., Alados-Arboledas, L., Bleisch, R., Huet, T., Ferrario, M. E., Madonna, F., Haefele, A., Nasir, F., Pace, G., and Posada, R.: A data assimilation experiment of temperature and humidity profiles from an international network of ground-based microwave radiometers, Proc. Microrad 2014, Pasadena, USA, 24-27 March, ISBN: 978-1-47994645-7, 978-1-4799-4644-0/14/\$31.00, 2014.

Cimini, D., Nelson, M., Güldner, J., and Ware, R.: Forecast indices from a ground-based microwave radiometer for operational meteorology, Atmos. Meas. Tech., 8, 315-333, doi:10.5194/amt-8315-2015, 2015.

Courtier, P., Andersson, E., Heckley, W., Vasiljevic, D., Hamrud, M., Hollingsworth, A., Rabier, F., Fisher, M., and Pailleux, J.: The ECMWF implementation of three-dimensional variational assimilation (3D-Var). I: Formulation, Q. J. Roy. Meteor. Soc., 124, 1783-1807, doi:10.1002/qj.49712455002, 1998.

Ding, S., Yang, P., Weng, F., Liu, Q., Han, Y., Van Delst, P., Li, J., and Baum, B.: Validation of the community radiative transfer model, J. Quant. Spectrosc. Ra., 112, 1050-1064, 2011.

Ebell, K., Orlandi, E., Hünerbein, A., Löhnert, U., and Crewell, S.: Combining ground and satellite based measurements in the atmospheric state retrieval: Assessment of the information content, J. Geophys. Res., 18, 6940-6956, 2013.

English, S. J., Poulsen, C., and Smith, A. J.: Forward modelling for liquid water cloud and land surface emissivity Proceedings of ECMWF workshop on ATOVS, ECMWF, Reading, UK, 25 November 1999.

Eriksson, P. and Buehler, S.: ARTS user guide, available at: http://www.radiativetransfer.org/misc/arts-doc-stable/uguide/ arts_user.pdf (last access: 1 March 2016), 2015.

Eriksson, P., Buehler, S. A., Davis, C. P., Emde, C., and Lemke, O.: ARTS, the atmospheric radiative transfer simulator, Version 2, J. Quant. Spectrosc. Ra., 112, 1551-1558, doi:10.1016/j.jqsrt.2011.03.001, 2011.

Eyre, J. R.: A fast radiative transfer model for satellite sounding 105 systems, ECMWF Technical Memorandum 176, ECMWF, Reading, UK, available at: http://www.ecmwf.int/sites/default/files/elibrary/1991/ 9329-fast-radiative-transfer-model-satellite-sounding-systems. pdf (last access: 1 March 2016), 1991.

Geer, A. J., Bauer, P., and Lopez, P.: Lessons learnt from the operational 1D 4DVar assimilation of rain and cloud affected SSM/I observations at ECMWF, Q. J. Roy. Meteor. Soc., 134, 15131525, 2008. 
Hewison, T.: 1D-VAR Retrievals of Temperature and Humidity Profiles From a Ground-Based Microwave Radiometer, IEEE TGRS, 45, 2163-2168, 2007.

Hewison, T. J., Cimini, D., Martin, L., Gaffard, C., and Nash, J.: Validating clear air absorption model using ground-based microwave radiometers and vice-versa, Meteorol. Z., 15, 27-36, 2006.

Hocking, J.: Interpolation methods in the RTTOV radiative transfer model, Forecast Research Technical Report No. 590, Met Office, Exeter, UK, available at: http://www.metoffice.gov.uk/ media/pdf/i/k/FRTR590.pdf (last access: 1 March 2016), 2014.

Hocking, J., Rayer, P., Saunders, R., Madricardi, M., Geer, A., Brunel, P., and Vidot, J.: RTTOV v11 Users Guide, Doc ID: NWPSAF-MO-UD-028, available at: https://nwpsaf.eu/ deliverables/rtm/docs_rttov11/users_guide_11_v1.4.pdf (last access: 1 March 2016), 2015.

Kneifel, S., Löhnert, U., Battaglia, A., Crewell, S., and Siebler, D.: Snow scattering signals in ground-based passive microwave measurements, J. Geophys. Res., 115, D16214, doi:10.1029/2010JD013856, 2010.

Lamkaouchy, K., Balama, A., and Ellison, W. J.: New permittivity data for sea water $(30-100 \mathrm{GHz})$, Extension of ESA report 11197/94/NL/CN, ESA, Paris, France, 1997.

Liebe, H. J., Hufford, G. A., and Manabe, T.: A model for the complex permittivity of water at frequencies below $1 \mathrm{THz}$, Int. J. Infrared and Millimetre Waves, 12, 659-671, 1991.

Liebe, H. J., Hufford, G. A., and Cotton, M. G.: Propagation modeling of moist air and suspended water/ice particles at frequencies below $1000 \mathrm{GHz}$, AGARD 52nd Specialists' Meeting of the Electromagnetic Wave Propagation Panel, Ch3, 1993.

Löhnert, U. and Maier, O.: Operational profiling of temperature using ground-based microwave radiometry at Payerne: prospects and challenges, Atmos. Meas. Tech., 5, 1121-1134, doi:10.5194/amt-5-1121-2012, 2012.

Löhnert, U., Turner, D., and Crewell, S.: Ground-Based Temperature and Humidity Profiling Using Spectral Infrared and Microwave Observations. Part I: Simulated Retrieval Performance in Clear-Sky Conditions, J. Appl. Meteorol. Clim., 48, 10171032, 2009.

Martinet, P., Dabas, A., Donier, J.-M., Douffet, T., Garrouste, O., and Guillot, R.: 1D-Var Temperature retrievals from Microwave Radiometer and convective scale Model, Tellus A, 67, 27925, doi:10.3402/tellusa.v67.27925, 2015.

Matricardi, M.: The inclusion of aerosols and clouds in RTIASI, the ECMWF fast radiative transfer model for the Infrared Atmospheric Sounding Interferometer, ECMWF Technical Memorandum 474, ECMWF, Reading, UK, available at: http://www.ecmwf.int/sites/default/files/elibrary/2005/11020inclusion-aerosols-and-clouds-rtiasi-ecmwf-fast-radiativetransfer-model-infrared-atmospheric.pdf (last access: 1 March 2016), 2005.
Matricardi, M.: The generation of RTTOV regression coefficients for IASI and AIRS using a new profile training set and a new line-by-line dataset, ECMWF Technical Memorandum 564, ECMWF, Reading, UK, available at: http://www.ecmwf.int/sites/default/files/elibrary/2008/11040generation-rttov-regression-coefficients-iasi-and-airs-usingnew-profile-training-set-and-new.pdf (last access: 1 March 2016), 2008.

Matricardi, M., Chevallier, F., and Tjemkes, S.: An improved general fast radiative transfer model for the assimilation of radiance observations, ECMWF Technical Memorandum 345, ECMWF, Reading, UK, available at: http://www.ecmwf.int/sites/default/files/elibrary/2001/11030improved-general-fast-radiative-transfer-model-assimilationradiance-observations.pdf (last access: 1 March 2016), 2001.

National Research Council: Committee on Developing Mesoscale Meteorological Observational Capabilities to Meet Multiple Needs, Observing Weather and Climate from the Ground Up, A Nationwide Network of Networks, ISBN: 978-0-309-129862, 250 pp., 2008.

NWPSAF: RTTOV v11, Met Office, Exeter, UK, available at: http: //nwpsaf.eu/site/software/rttov/rttov-v11/ (last access: 1 March 2016), 2013.

Rose, T., Crewell, S., Löhnert, U., and Simmer, C.: A network suitable microwave radiometer for operational monitoring of the cloudy atmosphere, Atmos. Res., 75, 183-200, 2005.

Rosenkranz, P. W.: Water vapor microwave continuum absorption: A comparison of measurements and models, Radio Sci., 33, 919928, doi:10.1029/98RS01182, 1998.

Saunders, R.: RTTOV-7 Science and validation report, available at: https://nwpsaf.eu/deliverables/rtm/rttov7_svr.pdf (last access: 1 March 2016), 2002.

Saunders, R.: RTTOV-9 Science and validation report, Doc ID: NWPSAF-MO-TV-020, available at: https://www.nwpsaf.eu/ deliverables/rtm/rttov9_files/rttov9_svr.pdf (last access: 1 March 2016), 2010.

Saunders, R. W., Matricardi, M., and Brunel, P.: An Improved Fast Radiative Transfer Model for Assimilation of Satellite Radiance Observations. Q. J. Roy. Meteor. Soc., 125, 1407-1425, 1999.

Seity, Y., Brousseau, P., Malardel, S., Hello, G., Benard, P., Bouttier, F., Lac, C., and Masson, V.: The AROME-France convectivescale operational model, Mon. Weather Rev., 139, 976-991, 2010.

Weston, P.: NWPSAF 1D-Var User Manual, Met Office Doc ID: NWPSAF-MO-UD-032, Met Office, Exeter, UK, 2014.

WMO: Statement Of Guidance For Global Numerical Weather Prediction, available at: http://www.wmo.int/pages/prog/www/ OSY/SOG/SoG-Global-NWP.pdf (last access: 1 March 2016), 2014. 